\title{
Object file continuity predicts attentional blink magnitude
}

\author{
FRANCES J. KELLIE and KIMRON L. SHAPIRO \\ University of Wales, Bangor, Wales
}

\begin{abstract}
When asked to identify targets embedded within a rapid consecutive stream of visual stimuli, observers are less able to identify the second target (T2) when it is presented within half a second of the first (T1); this deficit has been termed the attentional blink (AB). Rapid serial visual presentation methodology was used to investigate the relationship between the $\mathrm{AB}$ and object files (episodic representations implicated in object identification and perceptual constancy). An inverse linear relationship was found between the degree of object file continuity and $\mathrm{AB}$ magnitude. An important locus of object file continuity was the intervening stream items between $\mathrm{T} 1$ and $\mathrm{T} 2$. The results are discussed in terms of the heuristic of the object file to preserve limited attentional capacity.
\end{abstract}

Dynamic visual information that reaches the retinae during natural vision is intrinsically varied in wavelength and luminance across both space and time. When faced with such a complex stream of information, the brain must face processing limitations if it is to formulate accurate perceptions and instigate appropriate behavioral responses. Such temporal processing limitations can be examined empirically by using rapid serial visual presentation (RSVP). RSVP methodology uses stimuli (such as letters, words, pictures, digits, or sentences) that are typically presented in one spatial location in a rapid consecutive sequence (between 6 and 20 items/sec). A typical RSVP stream is displayed in Figure 1. Within the stream of stimuli, one item can be designated as the first target (T1), and this is followed by subsequent targets (T2, T3, etc.). Target items will differ in some way (e.g., color or identity) from all other items in the stream. Participants are required to identify (or detect) T1 and T2 (the dualtask condition) or to ignore $\mathrm{T} 1$ and just identify (or detect) T2 (the single-task baseline condition). Such tasks allow the experimenter to examine the participants' per-

This article is based on the doctoral dissertation of the first author, who was supported by a PhD Studentship R00429834497 from the Economic and Social Research Council (ESRC) and an ESRC Postdoctoral Fellowship T026271138. Some of the data from Experiments 1 and 2 were featured in a paper presented by the second author at the 40th Annual Meeting of the Psychonomic Society, Los Angeles, November 1999. Data from Experiments 1, 2, and 6 were presented by the first author at the 10th Annual Workshop on Object Perception and Memory, Kansas City, November 2002. We are grateful to our reviewers, Mary C. Potter and Vince Di Lollo, for their helpful comments, and we express our thanks to Anne Hillstrom for her help and support with the early "morphing" studies. Correspondence concerning this article should be addressed to F. J. Kellie, Centre for Cognitive Neuroscience, School of Psychology, University of Wales, Bangor, Gwynedd LL57 2AS, Wales (e-mail: f.kellie@bangor.ac.uk).

Note-This article was accepted by the previous editorial team, headed by Neil Macmillan. formance in relation to the interval between targets by manipulating the serial position of subsequent targets in relation to $\mathrm{T} 1$. With this paradigm, despite use of a diverse range of stimuli and response formats, the behavioral outcome is now well documented: Presenting T2 in close temporal proximity to $\mathrm{T} 1$ has a deleterious effect on participants' ability to carry out the T2 task for targets presented within half a second of the first (Broadbent \& Broadbent, 1987; Lawrence, 1971; Raymond, Shapiro, \& Arnell, 1992; Reeves \& Sperling, 1986; Weichselgartner $\&$ Sperling, 1987). This deficit was termed the attentional blink (AB) by Raymond et al.

Raymond et al.'s (1992) original theoretical explanation of the $\mathrm{AB}$ was based on the notion of inhibition. It was suggested that the $\mathrm{AB}$ might reflect $\mathrm{T} 1$ processing's monopolizing attentional resources to such a degree that the processing of any T2 attributes is inhibited. Later, Shapiro, Raymond, and Arnell (1994) adopted an alternative theoretical position based on the notion that interference arises from items (i.e., T1, T2, and their respective masks) competing for retrieval from a visual short term memory buffer. In contrast, Chun and Potter (1995) have explained the AB in terms of a two-stage model. During the first stage, all RSVP stream items proceed in parallel to high levels of analysis (e.g., semantic and featural registration), but stimuli become available for report only when they have been processed by a second serial processing stage. By this account, the AB occurs because the second stage is still employed in processing $\mathrm{T} 1$ and, therefore, cannot process $\mathrm{T} 2$.

Another theoretical explanation of the $\mathrm{AB}$ is centered on the masks that accompany T1 and T2 and the notion of object substitution (Brehaut, Enns, \& Di Lollo, 1999; Enns \& Di Lollo, 1997; Giesbrecht \& Di Lollo, 1998; Seiffert \& Di Lollo, 1997). Although proponents of the object substitution model generally have supported the two-stage model of Chun and Potter (1995), they have tended to emphasize the term substitution, in preference to interruption or overwriting, in order to highlight the 


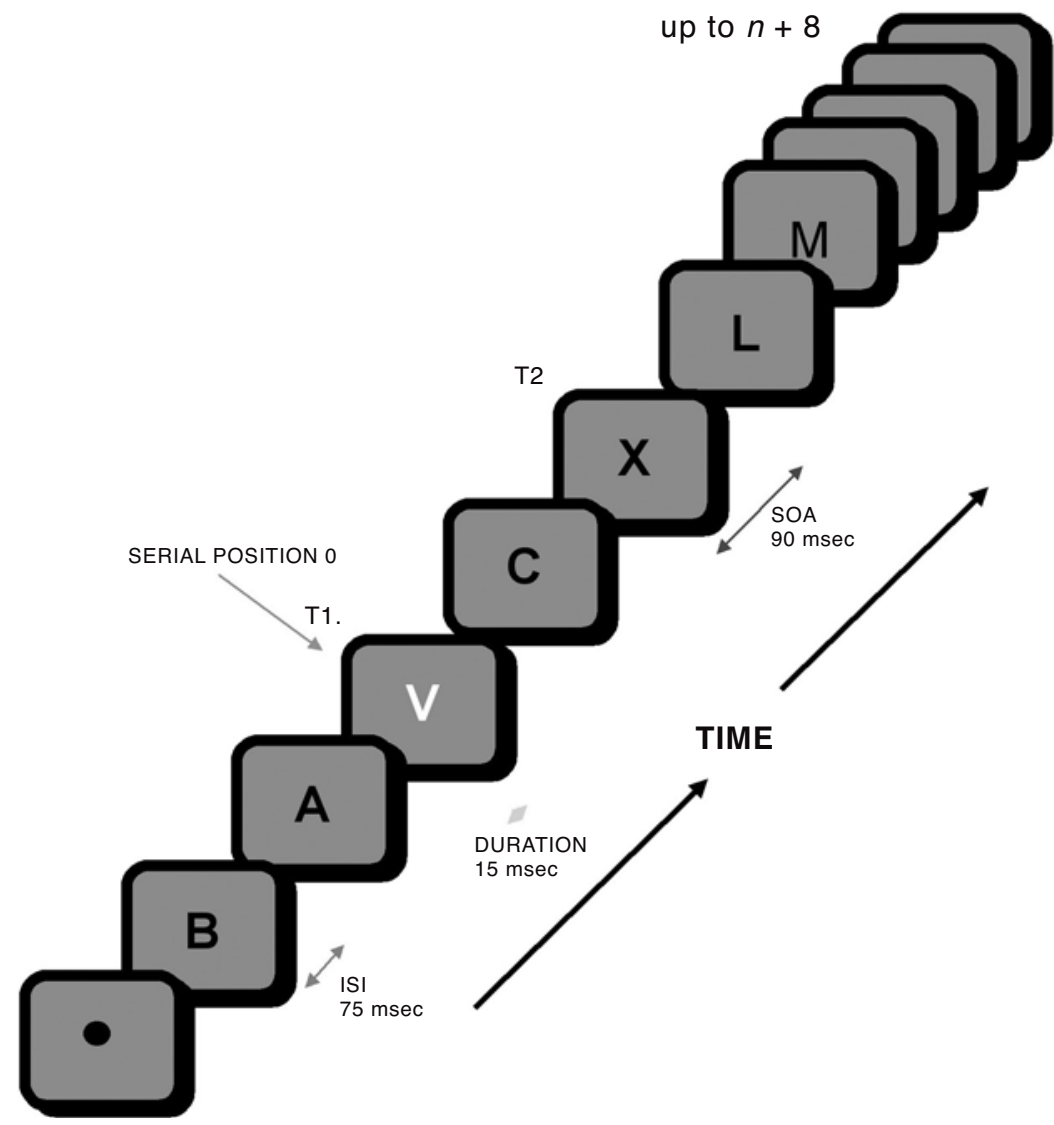

Figure 1. An example of a rapid serial visual presentation stimulus stream (as used by Raymond, Shapiro, \& Arnell, 1992, Experiment 2), in which participants were required to report the name of the white letter (T1) and detect whether a black X (T2) was present in the post-T1 stream. From "Temporary Suppression of Visual Processing in an RSVP Task: An Attentional Blink?" by J. E. Raymond, K. L. Shapiro, and K. M. Arnell, 1992, Journal of Experimental Psychology: Human Perception \& Performance, 18, p. 852. Copyright 1992 by the American Psychological Association. Adapted with permission.

function of the interruption mask that follows T2 (Brehaut et al., 1999; Enns \& Di Lollo, 1997; Giesbrecht \& Di Lollo, 1998). Enns and Di Lollo have suggested that the function of the mask is not merely to terminate target processing. Rather, the mechanisms involved in object recognition seem to turn their focus from processing $\mathrm{T} 2$ to processing its mask instead.

Although the aforementioned theories diverge in terms of the proposed mechanism underlying the $\mathrm{AB}$, Shapiro, Arnell, and Raymond (1997) have identified three central tenets that are common to all the theories mentioned here. First, the presence of a T1 mask means that increased attentional resources are required in order for $\mathrm{T} 1$ to be processed to the level of report. Second, a consequence of this is that there are fewer resources available for processing T2. Therefore, because T2 cannot attain a durable representation facilitating report, it is at risk of decay or object substitution (i.e., by T2's mask). However, this is resolved within approximately $500 \mathrm{msec}$ as the identity of $\mathrm{T} 1$ becomes consolidated. Third, in dis- tinctly specific conditions, response selection factors will have a further detrimental effect on T2 accuracy, resulting from additional pressures imposed on attentional capacity (such as a requirement for speeded responses).

Empirical evidence supports the proposition that objects serve an important function within the perception of visual scenes (Gordon \& Irwin, 1996). For example, Duncan (1984) presented participants with either a large or a small rectangle with a gap on either the right or the left side. Superimposed onto the rectangle was a dotted or dashed line that could be slanted toward the left or the right. The participants were required to make two judgments about the same object (e.g., large rectangle, gap on right) or to make two judgments about different objects (e.g., gap on right, dashed line). Duncan found that when the participants were asked to make judgments about different objects, they were less accurate. Similarly, Baylis and Driver (1993) and Watson and Kramer (1999) also found benefits associated with making oneobject judgments over those involving two objects. 
If objects play an important role in visual perception, there is also a necessity for a mechanism that serves to maintain object information over time (Gordon \& Irwin, 1996). The need for such a mechanism is illustrated in perceptual constancy: When we make eye movements or when objects move, despite the resulting changes in the size, shape, and position of the retinal image, we retain a sense of perceptual continuity, rather than reperceive the objects as novel ones (Gordon \& Irwin, 1996). Although the true nature of such a mechanism is not known, one possible candidate is the notion of the object file proposed by Kahneman, Treisman, and Gibbs (1992). Object files are episodic representations of objects in the real world (Kahneman et al., 1992). Being temporary in nature, object files are thought to be distinct from more durable, long-term (type) representations used to classify and identify objects. Theory suggests that an object file is created when we direct our attention to an object in the visual field. This file then collects and maintains information relating to that object and might possibly include all of its defining information, such as identity (if it is known) and meaning.

The object file reviewing process involves accessing an active object file, retrieving the information about object attributes, and establishing whether it matches the object currently in view. Object continuity persists when the information matches. In contrast, when information is incongruent across successive displays, this necessitates making modifications to the active object file or discarding it and generating a new object file. In both cases, object identification will be slowed (as compared with cases in which the same object file can be used), because object file operations are time consuming, presumably because of demands placed on attentional resources (Kahneman et al., 1992).

It can be seen that in the $\mathrm{AB}$, there are costs associated with dividing one's attention between two targets in a temporal stream for about $500 \mathrm{msec}$. Similar costs have also been seen in object file research, in terms of slower object recognition times when successive visual displays do not easily share a common object file. It might, therefore, be possible to attenuate the $\mathrm{AB}$ effect if $\mathrm{T} 1$ and $\mathrm{T} 2$ share a common object file. To phrase this another way, could the attentional costs observed in the $\mathrm{AB}$ be eliminated if attention is divided within an object (or object file), rather than between objects (or object files)? A recent study by Raymond (2003) lends support to such a notion.

Raymond (2003) used RSVP streams consisting of trident distractor stimuli presented in varying orientations to induce the percept of a single rotating trident. T1 was a thick bar that could occur on a trident stimulus (sameobject condition) or on an arrow (different-object condition) or could not appear at all. Participants were required to report the absence or presence of $\mathrm{T} 1$ and, if $\mathrm{T} 1$ was present, report whether it was on a trident or on an arrow. For T2 (which was present on half of the trials), participants had to detect a short line attached to either a trident (Experiment 1) or an arrow (Experiment 2). In the same-object condition, in which $\mathrm{T} 1 \mathrm{~s}$ were features of a single object (the trident), the AB effect was completely attenuated. In contrast, in the different-object condition (i.e., an arrow), an $\mathrm{AB}$ effect was obtained. To explain this, Raymond suggested that the object file, which was initially established when the first distractor was presented, could be easily updated and utilized for T1 when it was on the same object (trident), but when T1 appeared on a new object (an arrow), the existing object file could not be easily updated. Instead, the file had to be discarded, and a new object file had to be opened. Since this process is attentionally demanding, the update file process (which would be required to perform the $\mathrm{T} 2$ detection task) might be less efficient until such time as the open file operation was complete (Raymond, 2003).

The experiments reported here followed from and expanded Raymond's (2003) conclusions by examining the degree of object file continuity provided by the RSVP streams, in order to examine its impact on AB magnitude. The contribution of various aspects of the stream toward object file continuity and $\mathrm{AB}$ modulation was also examined.

\section{GENERAL METHOD}

\section{Design}

Factorial designs were used for this series of experiments. The dependent variable was RSVP task performance expressed as AB magnitude. The percentage of correct identifications of $\mathrm{T} 2$ for each serial position of T2 when T1 was correctly identified was calculated for the dual-task condition. In the single-task condition, the number of correct $\mathrm{T} 2$ identifications was expressed as a percentage of the total number of single-task trials for each serial position of $\mathrm{T} 2$. To obtain AB magnitude, T2 performance in the dual-task condition was subtracted from that observed in the single-task condition for each participant at each stimulus onset asynchrony (SOA). We are grateful to our reviewers for pointing out the need to demonstrate that $\mathrm{AB}$ magnitudes (attenuated or otherwise) result from an $\mathrm{AB}$ effect in the dual-task condition, rather than being artifacts of performance in the single-task condition. For this reason, single and dual performances are shown graphically (panel B) alongside each figure displaying $\mathrm{AB}$ magnitude (panel $\mathrm{A}$ ).

\section{Participants}

All the participants were adults with normal (or corrected) vision. The participants were recruited from the student participant panel and the community participant database and, for a 90-min session, received course credit or $£ 7$, respectively.

\section{Apparatus}

Stimuli were presented using RSVP custom software on an Apple Power Macintosh 8600/200 computer. The stimuli were viewed on an Apple 28-cm RGB monitor, using binocular vision from a viewing distance of $60 \mathrm{~cm}$. Illumination in the room was reduced during testing. The participants entered their responses into the computer via the keyboard.

\section{Stimuli and Procedure}

In order to generate RSVP streams, a morph movie of a smoking pipe changing into a saucepan was created using Morph Version 1.1 software. The movie was then dissected into 24 frames, using Convert QuickTime Movie to PICT Version 2. Specific frames were im- 
ported into Adobe Photoshop Version 5.0, and texture effects were applied in order to create the target stimuli. To create T1 images, the 12 th movie frame was modified so that it consisted entirely of either large dot elements (using pointillist effect, size 6) or small dot elements (pointillist effect, size 3). A different texture effect (patchwork) was used to create T2 stimuli that consisted of either large squares (patchwork effect, size 4/5) or small squares (patchwork effect, size 1/5). T2 images were adapted from Movie Frames 13-20. Example target stimuli are shown in Figure 2. Despite changing the textural nature of the elements that made up the targets, the spatiotemporal and color characteristics of the images from which they were derived were relatively preserved.

RSVP streams were displayed at a single location - the center of a subtended $\left(18.5^{\circ} \times 17.1^{\circ}\right)$ gray field of uniform luminosity. The visual angles subtended by the stimuli varied. Images from the morph movie subtended $2.96^{\circ}$ in height and $5.71^{\circ}$ in width. The visual angles subtended by the random-object distractors (where used) varied in height (from $7.13^{\circ}$ to $0.95^{\circ}$ ) and width (from $6.18^{\circ}$ to $2.39^{\circ}$ ).

In any given trial, T2 never preceded T1, and no frame could appear more than once. All the participants undertook blocks of singletask, as well as dual-task, trials. In single-task trials, they were required to ignore T1 and attend to and report only T2. In dual-task trials, the participants were required to attend to and report both targets. The order of the blocks was counterbalanced in terms of task type (i.e., single- or dual-task trials). When commencing the RSVP trials, the participants were instructed to attend to a center black fixation dot where the stimulus stream would subsequently be presented. All RSVP trials were self-paced and initiated by the participant by depressing the space bar. The participants were instructed to watch each RSVP stream and, once the stream finished, make their keyboard response(s) by pressing keys that had previously been labeled big and small. For the dual-task trials, the participants were instructed to make their first response in relation to the first target (i.e., dots) and their second response in relation to the second target (i.e., squares).

The participants were informed that accuracy, and not reaction time, was being measured. Before the experimental trials commenced, the participants were shown A4 color printouts of the target stimuli, and then three sets of practice trials were conducted. The first set consisted of 16 dual-task trials (4 trials at each of T2 Serial Positions 5-8, corresponding to SOAs of 333, 400, 467, and $534 \mathrm{msec}$ ) presented at the slower rate of $133.33 \mathrm{msec}$ per stimulus ( 8 items per second). The second set consisted of 16 dual-task trials presented using the same timings but utilizing the earlier T2 serial positions (i.e., 4 trials at each of Serial Positions 1-4, corresponding to SOAs of $67,133,200$, and $267 \mathrm{msec}$ ). The third practice set involved completing one block of 32 dual-task trials ( 4 trials at all SOAs) at the faster presentation rate of $66.67 \mathrm{msec}$ per stimulus (15 items per second). Each participant then undertook 320 experimental trials (one set of 160 single-task trials and one set of 160 dual-task trials). For those experiments utilizing seven SOAs rather than eight (Experiments 3, 4, 5, and 6), RSVP streams for the 67-msec SOA were removed from the training and experimental trials. Hence, Training Set 2 was reduced to 12 trials, Training Set 3 was reduced to 28 trials, and the number of experimental trials was reduced to 240 (one set of 140 single-task trials and one set of dualtask trials). No blank interstimulus interval (ISI) was presented between the stimuli.

Given that there were two possible T1 responses (big/small dots) and two possible $\mathrm{T} 2$ responses (big/small squares), this yielded four possible target combinations for each of the eight T2 serial positions ( 32 trials). Each combination was repeated five times, resulting in sets of 160 trials. This yielded 20 trials at each SOA. Experimental sessions lasted between $60 \mathrm{~min}$ (for streams without items appearing before T1) and $90 \mathrm{~min}$ (for full-stream experiments). The participants were replaced if their performance failed to meet criterion. Since $50 \%$ was chance (i.e., the target could be either big or small), these criteria were set at a mean of $50 \%$ correct for T 1 performance in the dual-task trials and for T2 performance in the singletask trials. Mean performance refers to performance across all SOAs.

\section{EXPERIMENT 1}

In Experiment 1, we sought to attenuate the $\mathrm{AB}$ through the linking of targets in an RSVP stream to a common object file. Earlier, it was noted how object files serve to maintain information about objects as they move or change over time (Kahneman et al., 1992). Whereas Raymond (2003) utilized object file information pertaining to an object's motion over time, the object file information maintained in the present experiments related to the object's changing identity over time and, in this way, makes an important contribution to our understanding of the way in which object files mediate divided attention.
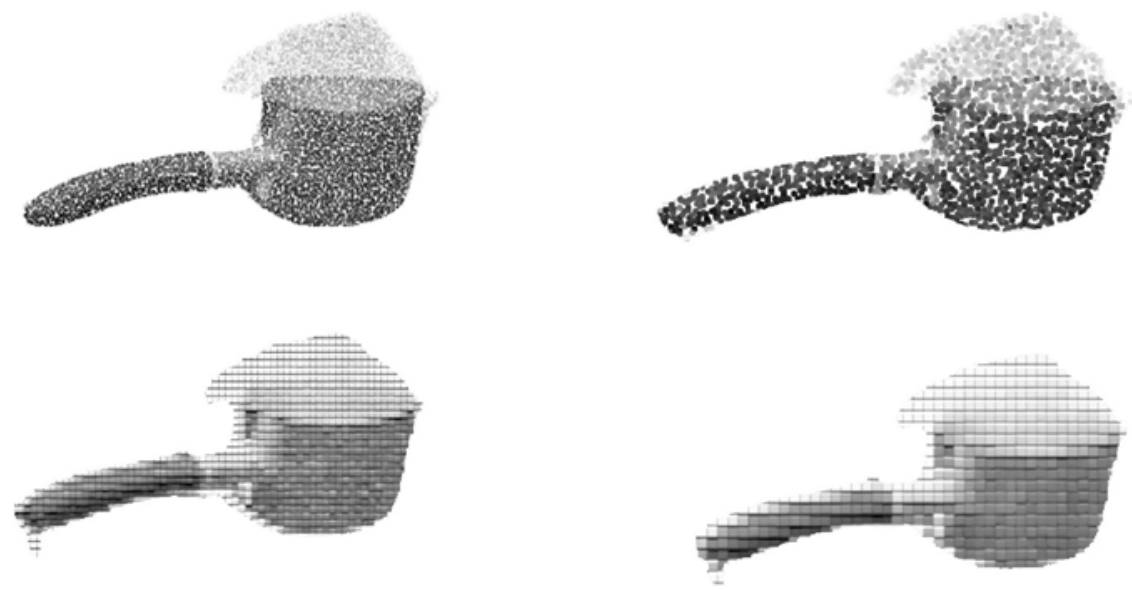

Figure 2. Rapid serial visual presentation targets adapted from the morph movie. T1 targets (shown top left and right) were either small or big dots. T2 targets (shown bottom left and right) were either small or big squares. 
In order to achieve this, a short movie was used in which a smoking pipe was seen to progressively change into a saucepan - a process termed morphing. If one adopts the same rationale as Raymond (2003), T1 should adopt the currently active object file by virtue of the preceding movie frames. In the morph RSVP stream in which one object changes smoothly into another, each successive frame should be so similar (in terms of spatiotemporal attributes) that the existing object file (the one generated at the start of the stream) should be utilized for each subsequent frame, including T1. If so, attentional resources would not be required to generate a new object file for T1, and therefore, T2 processing should not be hindered by $\mathrm{T} 1$ interference (which would arise from attentionally demanding processes, such as making extensive modifications to object files or generating new ones). Consequently, it was anticipated that an attenuated $\mathrm{AB}$ effect would be obtained in the morph condition.

A scrambled morph RSVP stream is a good control condition, because it uses the same stimuli as the smooth morph stream but presents all of the frames, apart from the targets, in a random order. In this condition, there is no longer a percept of smooth object change, and it was hypothesized that object file continuity would be disrupted and an $\mathrm{AB}$ would be observed.

\section{Method}

Design. In this experiment, an $8 \times 2$ mixed factorial design was employed. The within-groups factor was the period of time between the onsets of T1 and T2 (SOA), which had eight levels (67, 133, 200,
$267,333,400,467$, and $534 \mathrm{msec}$ ). The between-groups factor was the nature of the RSVP streams (stream), which had two levels (morph, where successive stream items gave the percept of a smoking pipe changing into a saucepan, and scrambled, where the morph movie frames were presented in a random order about T1 and T2).

Participants. Sixteen volunteers were recruited from the student participant panel (5) and the community participant panel (11). The participants were allocated randomly to the morph ( 5 females and 3 males; mean age, 23.6 years, $S D=6.23$ years) and scrambled conditions ( 6 females and 2 males; mean age, 24.5 years, $S D=6.74$ years).

Stimuli and Procedure. An example RSVP stream for each condition is shown in Figure 3. The participants were required to identify the size (big or small) of the dots for T1 and the size (big or small) of the squares for $\mathrm{T} 2$.

\section{Results and Discussion}

The mean percentage of correct $\mathrm{T} 1$ responses was significantly higher in the smooth morph condition $(97 \%$, $S D=5 \%$ ) than in the scrambled morph condition $[89 \%$, $S D=12 \% ; t(126)=4.728, p<.01]$. Mean AB magnitudes were calculated for each SOA and for both stream types. These are displayed in Figure 4 (panel A), which suggests that the proportion of correct $\mathrm{T} 2$ responses was reduced for the dual task, relative to the single task, at early SOAs for the scrambled condition, but not for the morph condition. That is, there appears to be an $\mathrm{AB}$ in the scrambled condition, but not in the morph condition. A two-way mixed (SOA, within subjects; stream, between subjects) analysis of variance (ANOVA) yielded a significant first-order interaction confirming a difference

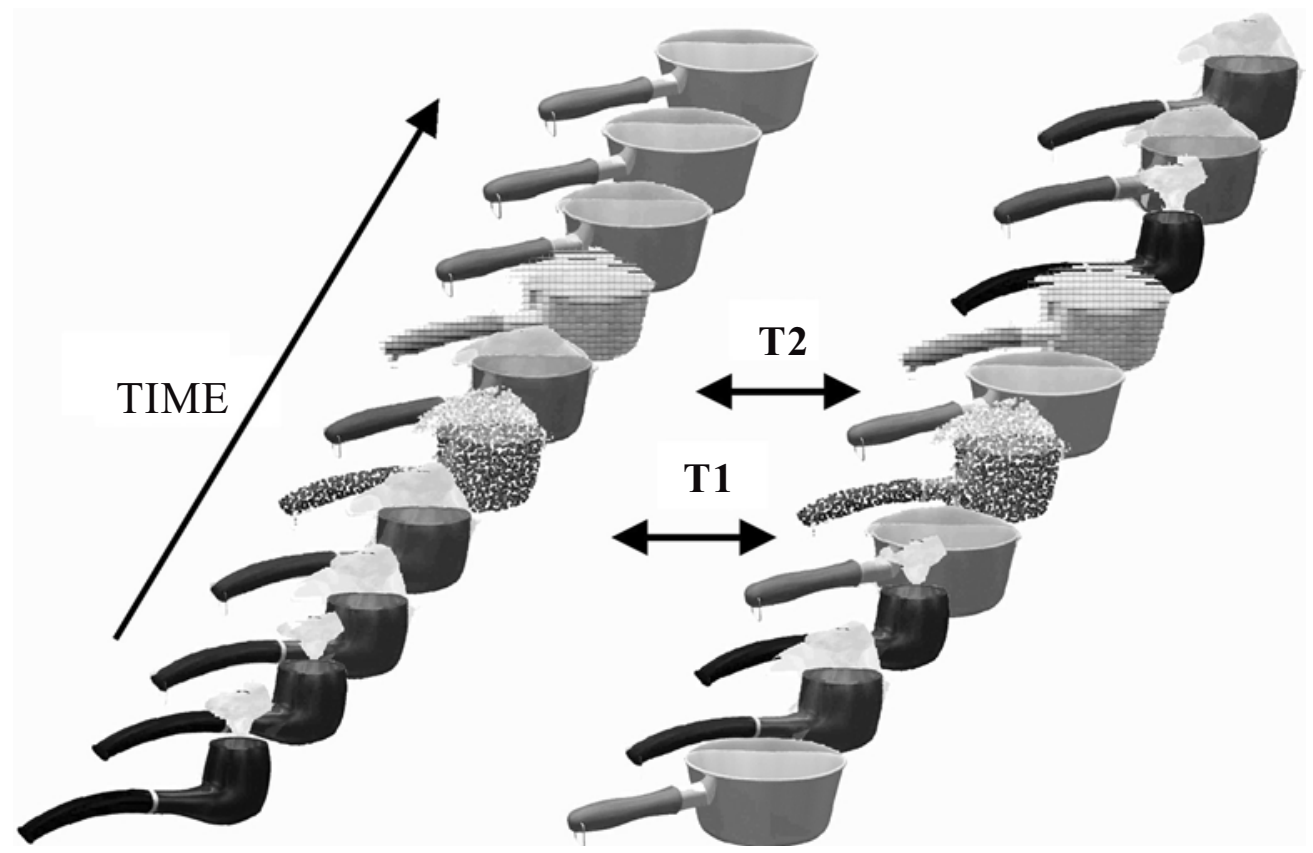

Figure 3. Rapid serial visual presentation streams for the smooth morph (shown on the left) and the scrambled morph (shown on the right). Streams were 24 frames long but have been truncated here for practical purposes. T2 is shown at Serial Position 2, which corresponds to a stimulus onset asynchrony of 133 msec. 


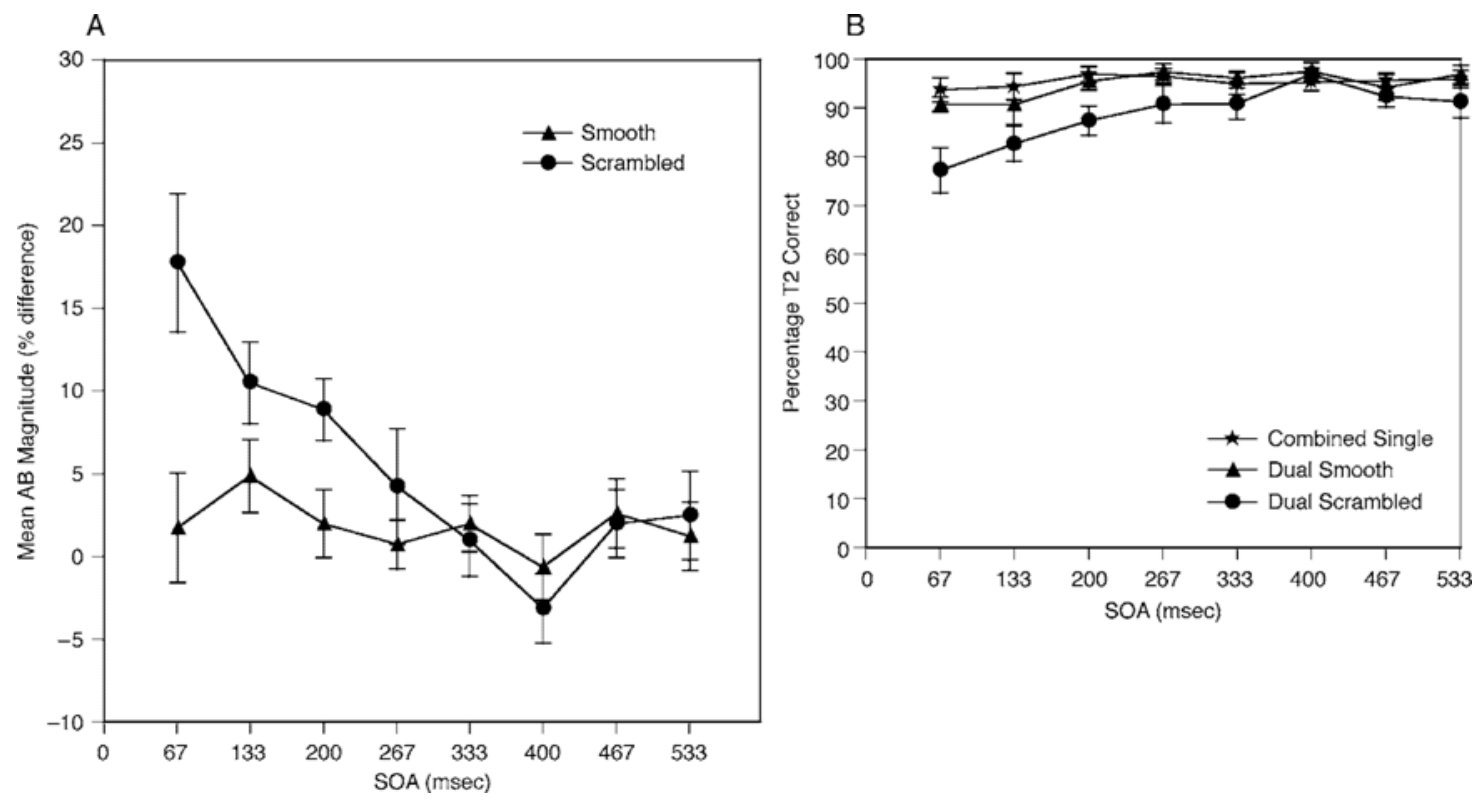

Figure 4. (A) Mean attentional blink (AB) magnitude for each stimulus onset asynchrony (SOA) for smooth and scrambled morph streams. Standard error $( \pm 1)$ is represented by the vertical bars. The same data are displayed in an alternative way in panel $B$, which depicts $T 2$ performance for smooth and scrambled morph streams for the dual-task trials. The single-task baseline reflects combined performance in the ignore T1 condition for the smooth and scrambled morph streams.

between the two stream types across SOAs $[F(7,98)=$ $3.127, p<.05]$. Similarly, significant main effects were observed for stream $[F(1,14)=6.125, p<.05]$ and SOA $[F(7,98)=4.903, p<.05]$.

Repeated measures ANOVAs were conducted on the $\mathrm{AB}$ magnitude data for all SOAs for both the scrambled and the smooth stream types, to ascertain whether a significant $\mathrm{AB}$ was present in each condition. As is predicted by the pattern of data in Figure 4, these analyses confirmed that there was no effect of SOA in the smooth morph stream, in which object file continuity was preserved $[F(7,49)=0.500, p>.05]$, but a significant effect of SOA in the scrambled stream, in which object file continuity was disrupted $[F(7,49)=6.748, p<.05]$. Furthermore, Figure 4 suggests that this $\mathrm{AB}$ effect persisted until $267 \mathrm{msec}$. To investigate this, single-sample $t$ tests (one-tailed) were carried out for the first four SOAs, and the alpha level was set to .01, a conservative level, to reflect the number of comparisons made. These tests demonstrated that the $\mathrm{AB}$ effect in the scrambled morph condition was significant at $67 \mathrm{msec}[t(7)=4.244, p<.01]$, $133 \mathrm{msec}[t(7)=4.274, p<.01]$, and $200 \mathrm{msec}[t(7)=$ $4.782, p<.01]$ but had recovered by $267 \mathrm{msec}[t(7)=$ $1.220, p>.01]$.

In the scrambled morph condition, despite the inherent disruption in object file continuity, each successive frame was generally similar in terms of low-level spatial information. It is possible that the shortened $\mathrm{AB}$ effect observed in this condition reflected minimal object file modifications, but greater modifications than those necessitated by the smooth morph condition. Since Kah- neman et al. (1992) claimed that greater attentional resources are implicated in generating new object files, one might, therefore, anticipate a larger $\mathrm{AB}$ effect (i.e., greater magnitude or longer temporal duration) when the stream requires more extensive object file changes to be made. One way to increase the disruption of object file continuity (and possibly create the demand for new object files) is to embed the same targets within a consecutive stream of random objects having spatial properties intrinsically different from those of the smoking pipe or the saucepan. This was examined in Experiment 2.

\section{EXPERIMENT 2}

In Experiment 1, AB attenuation was observed in the smooth morph condition, in which targets were able to share a common object file with minimal updating of object file information. In contrast, the scrambled morph condition necessitated greater changes to be made to the object file, and a significant (although shortened) AB effect was obtained. In Experiment 2, the same pipe-pan targets were embedded within an RSVP stream of random objects. It was hypothesized that a robust $A B$ effect would be obtained, since each successive frame was a new object and would demand a greater degree of object file modification (or the creation of new object files).

\section{Method}

The experimental method and procedure adopted were the same as those outlined in Experiment 1, with the exceptions described below. 
Design. A single factor, SOA (all eight levels), was employed in this experiment.

Participants. A total of 8 participants were recruited. The sample consisted of volunteers from the student participant panel (1 male and 3 females; mean age, 23.7 years, $S D=4.65$ years) and the community participant database (1 male and 3 females; mean age, 23.5 years, $S D=6.40$ years).

Stimuli and Procedure. In order to create RSVP streams for this experiment, the pipe-pan targets were embedded within a sequence of 22 pictures of random objects. An example stream is presented in Figure 5. As before, T1 appeared at Position 12 in the stream, and T2 could appear at one of the eight serial positions following $\mathrm{T} 1$.

\section{Results and Discussion}

The mean percentage of correct $\mathrm{T} 1$ responses in the dual-task trials was $87 \%(S D=12.07 \%)$. Mean $\mathrm{AB}$ magnitude was calculated for each SOA, and these are shown in Figure 6 (panel A), which suggests that an AB effect was obtained using a random-object RSVP stream. This was confirmed by an ANOVA that revealed a significant main effect of SOA $[F(7,49)=2.569, p<.05]$.

In order to establish the duration of the $\mathrm{AB}$ for the random-object stream, single-sample $t$ tests were conducted for each of the $\mathrm{AB}$ magnitudes between 133 and $400 \mathrm{msec}$, using a .01 alpha level to reflect the number of comparisons being made. Because of the increased variance (as compared with the scrambled morph stream in Experiment 1) and the modified alpha level, these

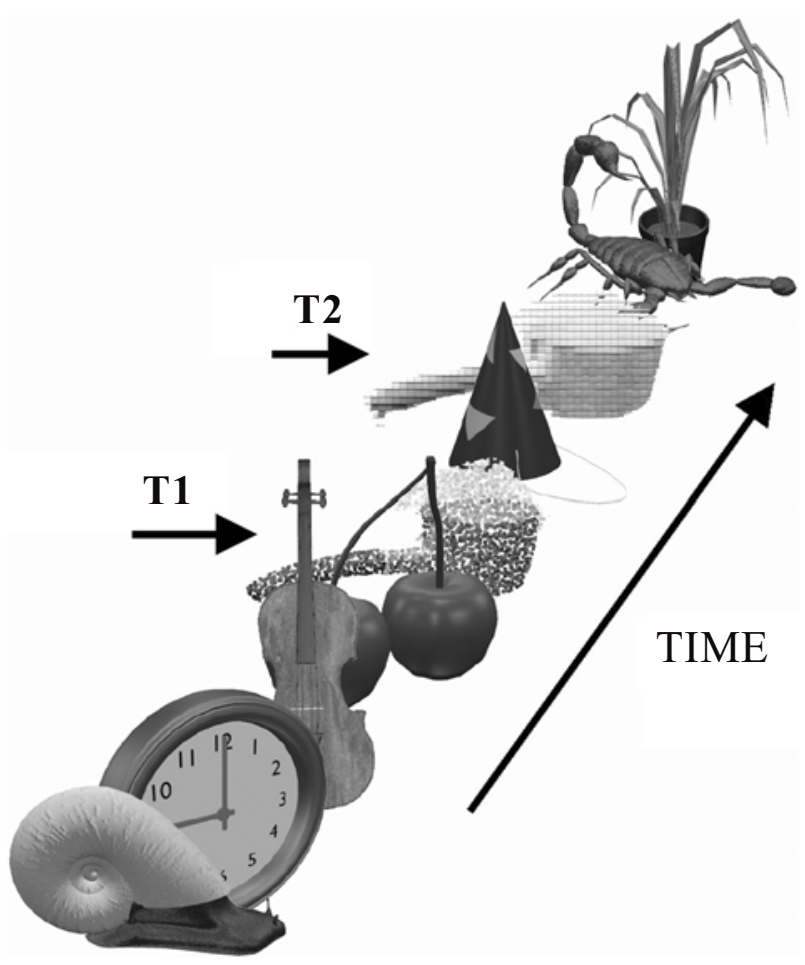

Figure 5. Example random object rapid serial visual presentation stream. Streams were 24 frames in length but have been truncated here for practical purposes. $T 2$ is shown in the second T2 serial position (corresponding to $133 \mathrm{msec}$ ). analyses were significant only for $267 \mathrm{msec}[t(7)=$ $3.380, p<.01]$ and $400 \mathrm{msec}[t(7)=4.241, p<.01]$. This demonstrates that the random-object stream yielded an $A B$ effect that did not recover until $467 \mathrm{msec}$. With this alpha level, the $\mathrm{AB}$ magnitudes at the following SOAs were not significant: $133 \mathrm{msec}[t(7)=1.699, p>.01]$, $200 \mathrm{msec}[t(7)=2.856, p>.01]$, and $333 \mathrm{msec}[t(7)=$ $2.700, p>.01]$. However, the single asterisks in Figure 6 demonstrate that if one adopts a less conservative alpha level (i.e., .05), the 200- and 333-msec SOAs would also have been significant.

In this experiment, we attempted to maximally disrupt object file continuity by utilizing random-object stimuli that should have initiated the attentionally demanding process of generating new object files. A robust $A B$ was observed, and this effect was longer in duration than that obtained in the scrambled morph condition in Experiment 1 . It is interesting to note that the onset of the $A B$ was not immediate - that is, lag-1 sparing was observed. This contrasts with the results in the scrambled morph condition in Experiment 1, where lag-1 sparing was not present.

\section{Discussion}

Experiments 1 and 2 successfully created optimum conditions for the preservation or disruption of object file continuity. In the smooth morph condition, in which object file (and perceptual) continuity was preserved, complete attenuation of the $\mathrm{AB}$ was observed. In contrast, a robust $\mathrm{AB}$ effect was associated with object file (and perceptual) discontinuity that was created by either the scrambled morph or the random-object stream.

The scrambled morph stream yielded an $\mathrm{AB}$ for the first three serial positions. This corresponded to $267 \mathrm{msec}$ and was a relatively short-lived effect, as compared with results in other studies that have reported an $\mathrm{AB}$ that continues until 450 msec (e.g., Raymond et al., 1992). Rather than reflecting the generation of new object files, this shortened AB effect may simply reflect a greater degree of object file updating than that required by the smooth morph stream.

It was anticipated that the object file discontinuity in the random-object stream would demand a greater degree of object file modification (or the creation of new object files, rather than the updating of existing ones). Since object file generation is thought to require greater attentional resources than does amending an object file (Kahneman et al., 1992), one would predict a larger AB (magnitude or duration) for the random-object stream than for the scrambled morph. This is supported by the fact that the $\mathrm{AB}$ effect in the random-object stream did not recover until $467 \mathrm{msec}$ after T1 onset (200 msec after $\mathrm{AB}$ recovery in the scrambled morph condition). Apart from differences in object file related factors, the RSVP streams used in Experiments 1 and 2 also differed in terms of the masks that followed T1 and T2. The importance of this factor in terms of the experimental outcomes was examined in Experiment 6. 


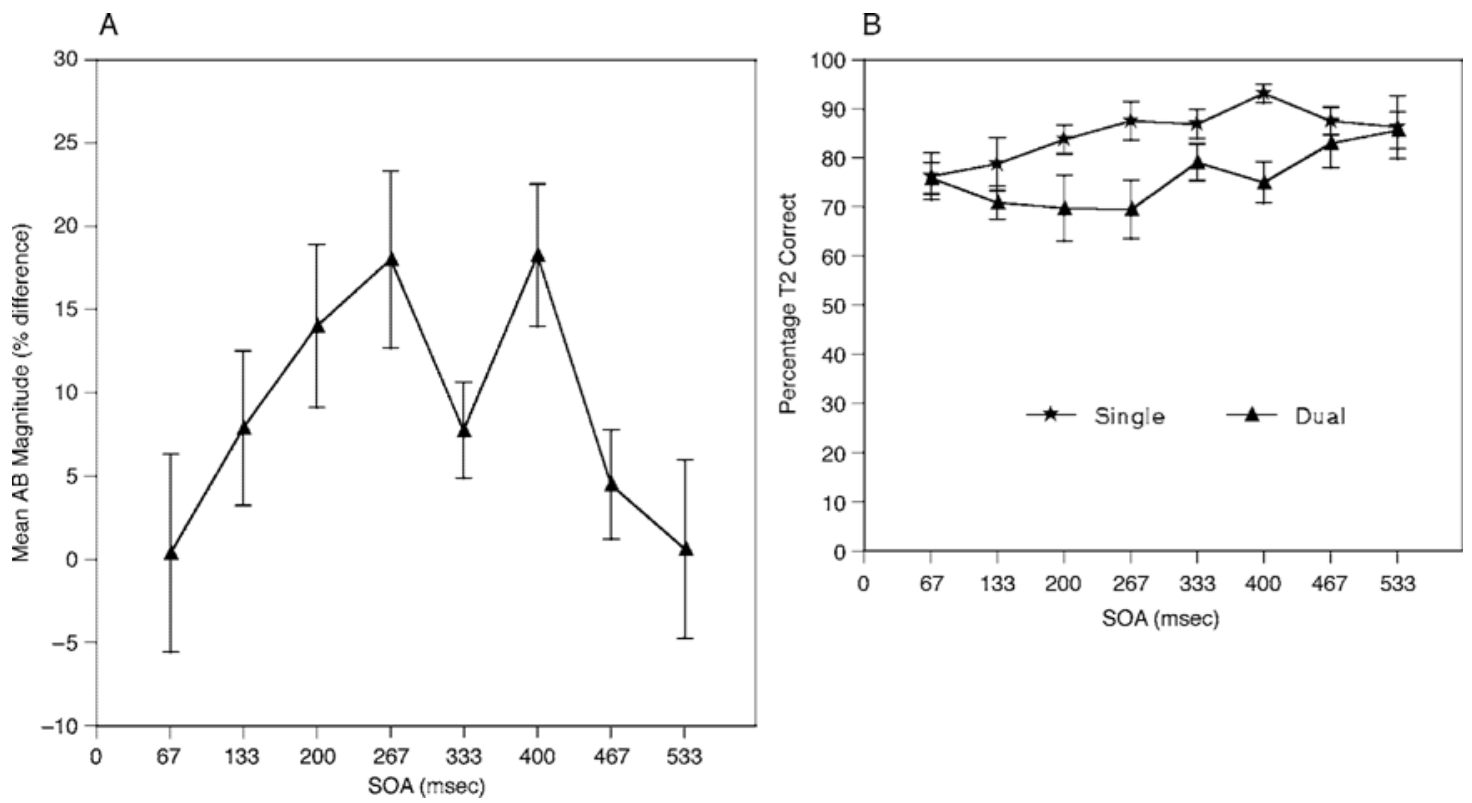

Figure 6. (A) Mean attentional blink (AB) magnitude for each stimulus onset asynchrony (SOA) for random-object stream. Standard error $( \pm 1)$ is represented by the vertical bars. The same data are displayed in an alternative way in panel $B$, which depicts $T 2$ performance for both single- and dual-task trials.

Generally, one of two different $\mathrm{AB}$ functions is observed, a U-shaped function or a monotonic function. A review of the recent literature showed that both patterns have been reported with roughly equal frequency (Visser, Bischof, \& Di Lollo, 1999). The scrambled morph condition produced a monotonic function in which the deficit was most pronounced at the first SOA and was reduced as a function of increases in SOA. In contrast, the random object morph produced a U-shaped function in which the onset of the $\mathrm{AB}$ was not immediate (i.e., there was lag-1 sparing) but was greatest for the second or third SOA.

Both Chun and Potter (1995) and Shapiro and Raymond (1994) have postulated that lag-1 sparing occurs because of a slow-closing attentional gate. In a similar vein, Sperling and Weichselgartner (1995) have proposed the concept of a discrete attentional episode, or window of attention, that can take up to $200 \mathrm{msec}$ to close. By this account, lag-1 sparing occurs when both targets form part of a single attentional window (or episode). However, if this is the case, one might expect it to be obtained consistently (Visser et al., 1999).

One possibility is that lag-1 sparing is a reflection of attentional switching - that is, how efficiently the system can, after processing $\mathrm{T} 1$, reconfigure itself in order to accommodate the different processing demands of $\mathrm{T} 2$ when it occurs in close temporal proximity (Visser et al., 1999). When attentional switching is rapidly achieved, there is no deficit in T2 performance at this SOA, and therefore, lag-1 sparing occurs. However, when the attentional-switching process is slow (possibly because the reconfiguration process was unable to cope with ex- cessive switching demands), T2 performance is impaired, and lag-1 sparing is not obtained.

In a review of over 100 experiments, Visser et al. (1999) attempted to identify those attentional-switching conditions associated with the presence or absence of lag-1 sparing. They concluded that lag-1 sparing is obtained when there is no attentional switch between targets or when there is a unidimensional switch between targets, such as a switch in task (e.g., identify T1, detect T2) or category (e.g., T1 letter, T2 digit). In contrast, when there is a switch in location (T1 and T2 appear in different spatial locations) or a multidimensional switch between targets is required, lag-1 sparing does not occur. This explanation does not explain the pattern of results for the scrambled morph and random-object streams. Both streams involve a unidimensional switch in category (from dots to squares), and one might predict lag-1 sparing in both conditions, yet it was present only in the random-object stream. In an attempt to explain this disparity, one needs to consider the nature of the distractor items in each stream. In the random-object stream, a perceptual filter for the pipe-pan stimulus will select T1. Since the item immediately following $\mathrm{T} 1$ (the +1 item) is usually a non-pipe-pan item (which will not fit the filter), it is not essential for the filter to close rapidly in order to limit interference from the +1 item. Because the filter is slow to close, T2 is opportunely processed (by virtue of sharing the same attentional window), and lag-1 sparing is obtained. In the scrambled morph, where all the stream items are similar, the same perceptual filter will need to close rapidly in order for $\mathrm{T} 1$ to be processed 
efficiently while minimizing interference from very similar +1 items. Because the filter closes rapidly, T2 is unlikely to gain entry, and lag-1 sparing will not occur. Thus, the similarity of distractor items may also play a role in determining the pattern of the $\mathrm{AB}$ and, in particular, the presence or absence of lag-1 sparing.

The results of these experiments are consistent with the conclusions drawn by Raymond (2003) and Kahneman et al. (1992). The first item in the RSVP stream creates an object file, which is reviewed for each subsequent visual event. Where T1 is the same object as the preceding item (i.e., in the smooth morph stream), it can easily utilize the current object file. Because attentionally demanding object file operations have not been required, there are sufficient resources to fully process $\mathrm{T} 2$, and consequently, no AB is observed. In contrast, when $\mathrm{T} 1$ cannot easily adopt the current object file (i.e., in the scrambled morph or random-object streams), object file modification is required, and the degree of attentional demand may vary according to the degree of object file modification required. Since these processes interfere with the processing of $\mathrm{T} 2$ at early serial positions, an $\mathrm{AB}$ is observed. Although the results of these experiments are in agreement with this conceptual model, they do not (nor were they designed to) isolate the locus of the effect as being specific to the object file relationship between $\mathrm{T} 1$ and the preceding item, as Raymond suggested. Alternative candidates for the locus of this effect include the object file relationship between the two targets and the nature of the items between the two targets, which are in the purview of the present experiments.

Having identified the extreme conditions under which an $A B$ can be shown to be attenuated, the next three experiments attempted to identify factors that modulated the $\mathrm{AB}$ effect in the smooth morph stream (Experiment 1), for which the $\mathrm{AB}$ was attenuated, and in the random-object stream (Experiment 2), for which a robust effect was obtained. The decision to investigate these two stream types was based on the assumption that they represent opposite extremes in terms of the degree of object file modifications required. The scrambled morph condition, which presumably involves an intermediate degree of object file modification, did not feature in these investigations. In Experiment 3, we examined the smooth morph stream and investigated whether the process of morphing was essential in providing object file continuity. In the other two experiments, in which we examined the randomobject stream, we considered the role of the items intervening between T1 and T2 (Experiment 4) and the items preceding T1 (Experiment 5).

\section{EXPERIMENT 3}

In this experiment, we investigated whether the $\mathrm{AB}$ attenuation observed in the smooth morph stream in Experiment 1 was modulated by the morphing process that provided object file continuity between $\mathrm{T} 1$ and $\mathrm{T} 2$. If so, one would expect the $\mathrm{AB}$ to return if object file continu- ity were removed and the intervening items between the two targets were replaced by a variable blank interval. This critical items RSVP procedure had already been employed to show that an $\mathrm{AB}$ can be obtained by using streams consisting of $\mathrm{T} 1, \mathrm{~T} 2$, and their respective masks and varying the blank interval between T1's mask and T2 (Duncan, Ward, \& Shapiro, 1994).

\section{Method}

Design. In this experiment, one factor was used: the seven SOAs of T2. Since T1's mask is crucial to the critical items paradigm, the first T2 SOA (67 msec) could not be used, because that position was always occupied by T1's mask.

Participants. Twelve volunteers ( 5 females and 7 males; mean age, 22.8 years, $S D=4.15$ years) were recruited from the community participant panel.

Stimuli and Procedure. The modified RSVP paradigm involved reducing the full pipe-pan stream to T1, T2, and their respective masks and manipulating the SOA by inserting a blank interval between T1's mask and T2. The mask item was the next consecutive item from the morph movie. A typical RSVP stream is illustrated in Figure 7. Due to a parameterization error, half of the participants completed 16 trials at each SOA, rather than 20 (112 trials in total).

\section{Results and Discussion}

For the dual-task trials, the percentage of correct T1 responses was $83 \%(S D=12.23 \%)$. This was lower and more variable than $\mathrm{T} 1$ performance in the smooth morph condition and was comparable with performance in the full-stream random-object condition. Mean $\mathrm{AB}$ magnitudes were computed for each SOA, and these are displayed in Figure 8 (panel A). A significant AB effect was not obtained $[F(6,66)=0.750, p>.05]$. Similarly, these results were not found to be significantly different from the full-stream smooth morph condition $[F(6,108)=$ $0.797, p>.05]$.

These results suggest that in the smooth morph fullstream condition (in Experiment 1), the morph items between the two targets were not crucial for providing object file continuity. It appears that a single-object file can be maintained so long as the visual information is not inconsistent with the object file reviewing process (see below). However, the greater degree of variability in this experiment (as compared with the morph condition in Experiment 1) suggests that the morphing process does contribute to perceptual continuity in some way.

The outcome of this experiment can be explained in terms of object file theory, which holds that when the current object file is reviewed, only the most recent visual information is used (Kahneman et al., 1992). That is, when it is decided whether the current visual display fits with the current object file, the visual information is compared with that immediately preceding it. Therefore, in this experiment, it is difficult to establish whether the pipe-pan items that intervened between $\mathrm{T} 1$ and $\mathrm{T} 2$ were necessary to provide $\mathrm{T} 1 / \mathrm{T} 2$ continuity, because the frames that were displayed were consistent with the current object file information. In addition, because the pipe-pan movie was 24 frames long, there was little object change 


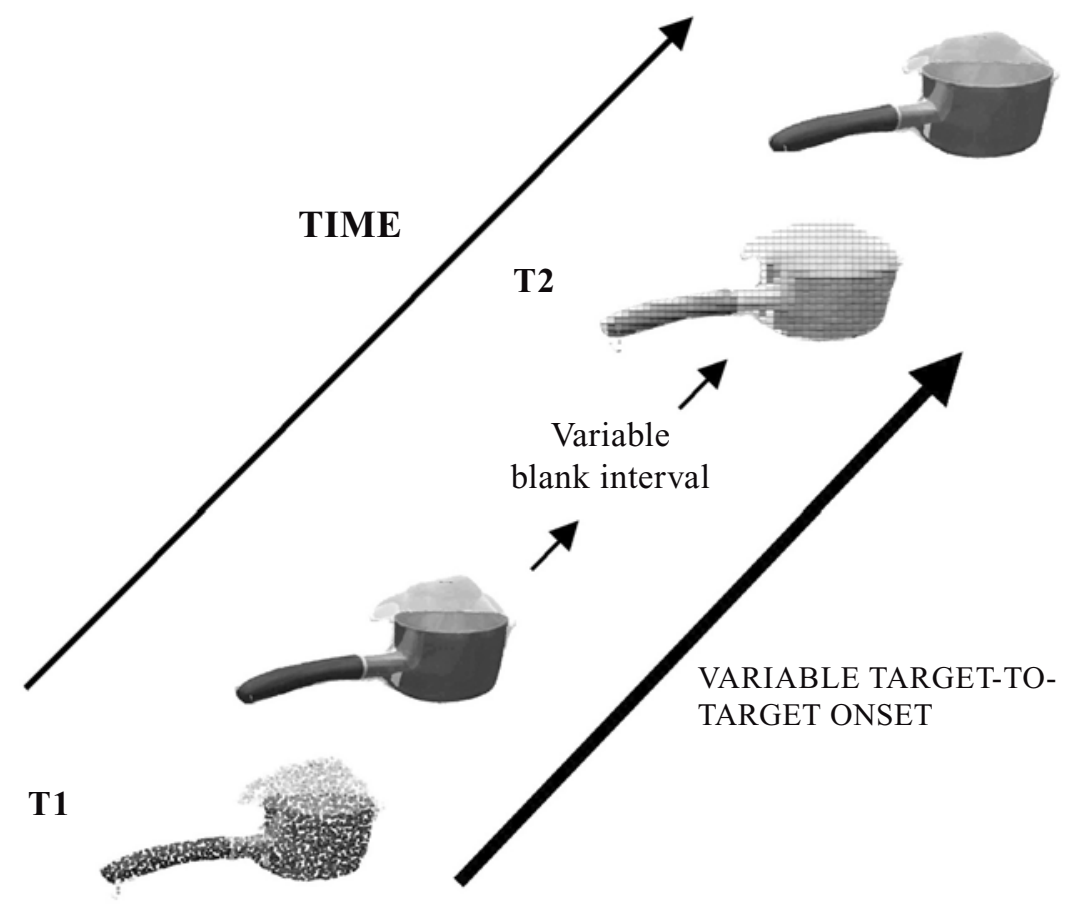

No items before $\mathrm{T} 1$

Figure 7. The critical items rapid serial visual presentation stream involved presenting $T 1, T 2$, and their respective masks (which were the next consecutive items from the morph movie). Variable target-to-target onset was provided by inserting blank frames between T1's mask and T2. As before, the participants were required to identify the size of dots (for T1) and the size of squares (for T2).

over three or four frames; removing the intervening items did not constitute much of a mismatch between the displayed frames.

\section{EXPERIMENT 4}

Given the outcome of Experiment 3, in Experiment 4, we further investigated Kahneman et al.'s (1992) notion of recency in object file reviewing and attempted to overwrite the T1 object information with that of a different object, so that T2 could not use the object file without making substantial modifications, which should theoretically result in an AB. This experiment was the same as Experiment 3, with the exceptions described below.

\section{Method}

Participants. Sixteen volunteers (12 females and 4 males; mean age, 21.6 years, $S D=3.76$ years) were recruited from either the community participant database (3) or the student participant panel (13).

Stimuli and Procedure. RSVP streams were the same as those in Experiment 3, with the exception that random objects were used to mask T1 and T2, rather than pipe-pan stimuli, and random objects were presented between T1's mask and T2. The number of random objects appearing in each stream ranged between one and seven (depending on SOA). A typical RSVP stream is illustrated in Figure 9.

Half of the participants completed their two sets of experimental trials in two sets. Set 1 consisted of 56 trials (yielding 8 trials at each SOA), and Set 2 comprised 84 trials (yielding 12 trials at each SOA). By splitting the trials into sets, it was possible to establish whether there were any overall effects from increased variability in the first part of the experimental trials (possibly due to unfamiliarity with the task). The remaining 8 participants completed two sets of 140 trials ( 20 trials at each SOA), as in the previous experiments. In this case, because of the way in which the morph program works, it was not possible to analyze the single-set data in terms of first and second halves, because the program randomly selects from the total number of trials (140), rather than from the total stream set (28), and consequently, an unequal number of trials at each SOA would be obtained if one were to split the data into two sets based on order of presentation.

\section{Results and Discussion}

The data from 2 participants were replaced because they made excessive T2 errors (54\% and 58\%) in the singletask trials. Before pooling the data, the data from participants who had completed two sets of trials were analyzed. Set 1 data appeared to be more variable than Set 2 data, and an ANOVA confirmed a significant main effect of set $[F(1,7)=7.981, p<.05]$. This suggests that during the first set, despite having completed 56 practice trials, the participants were still becoming accustomed to the task. Consequently, Set 1 data were removed, and only Set 2 data were pooled with the remaining data (where trials were completed in a single set). This reduced data 


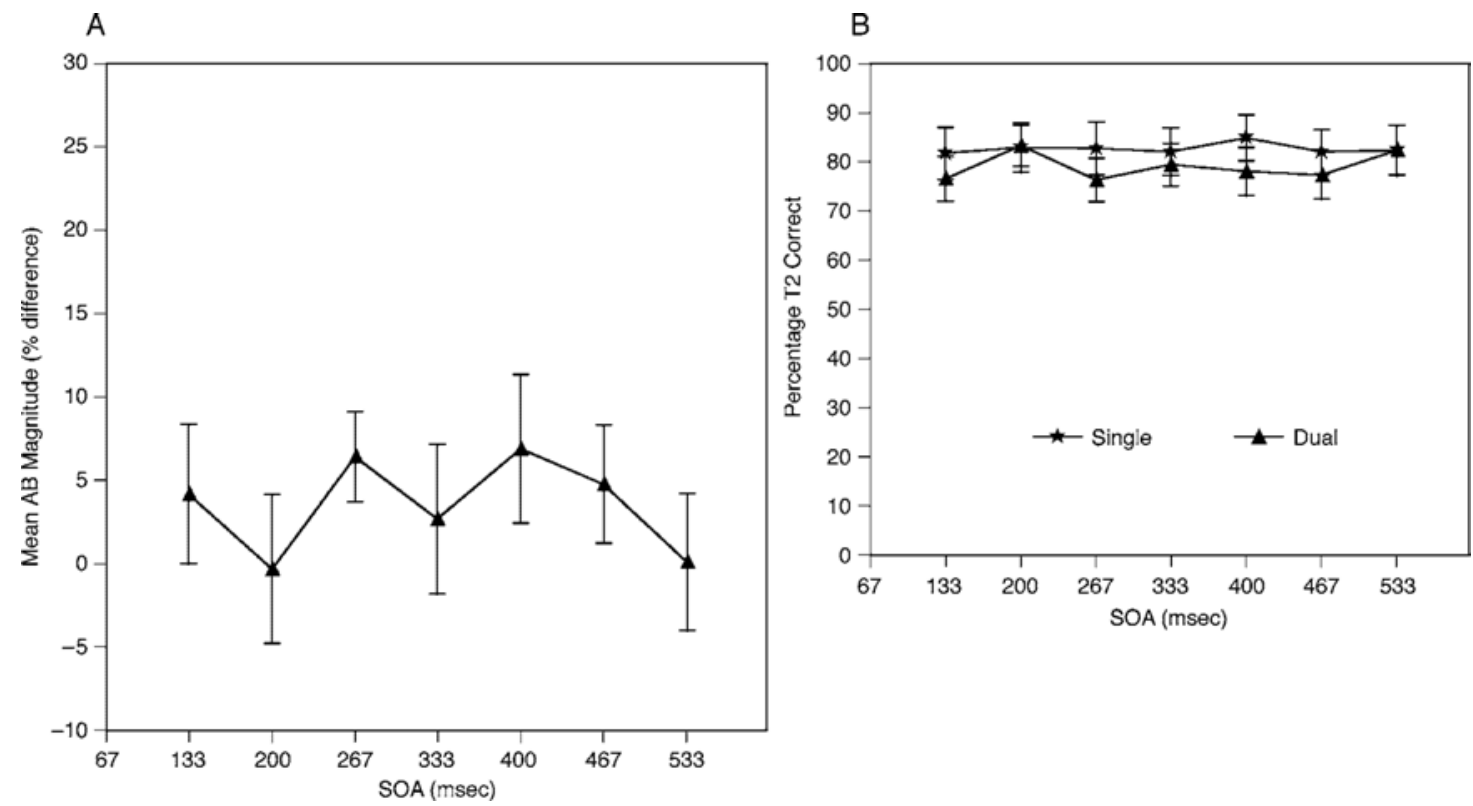

Figure 8. (A) Mean attentional blink (AB) magnitude for each stimulus onset asynchrony (SOA) for critical items stream, which involved the presentation of $\mathrm{T} 1, \mathrm{~T} 2$, and their respective masks. Standard error $( \pm 1)$ is represented by the vertical bars. The same data are displayed in an alternative way in panel B, which depicts $T 2$ performance for both single- and dual-task trials.

set was used for all subsequent analyses. The mean percentage of correct $\mathrm{T} 1$ responses in the dual-task trials was $84 \%(S D=17.32 \%)$. Mean AB magnitudes were calculated, and these are displayed in Figure 10 (panel A).

Figure 10 is suggestive of an $\mathrm{AB}$ effect. This was confirmed by an ANOVA, which revealed a significant effect of SOA $[F(6,90)=2.25, p<.05]$. These results are not significantly different from the original full stream of random objects $[F(6,132)=1.175, p>.05]$, which also yielded a significant AB. Single-sample $t$ tests (one-tailed) were conducted at all SOAs except 400 and $534 \mathrm{msec}$ (using a .01 alpha level). These analyses were significant for $133 \mathrm{msec}[t(15)=3.182, p<.01]$ and $267 \mathrm{msec}$ $[t(15)=4.654, p<.01]$. However, nonsignificant results were obtained for AB magnitudes at SOAs of $200 \mathrm{msec}$ $[t(15)=1.469, p>.01], 333 \mathrm{msec}[t(15)=1.489, p>$ $.01]$, and $467 \mathrm{msec}[t(15)=1.634, p>.01]$. If one were using the less conservative alpha level of .05 , these three SOAs would also approach significance.

These results are consistent with Raymond's (2003) theoretical framework. Since no items preceded T1, one would expect the presentation of T1 to initiate an open file process for each trial. This process consumes processing resources to the extent that $\mathrm{T} 2$ is unable to be processed to the level of report, and an $\mathrm{AB}$ effect would be anticipated.

In terms of Kahneman et al.'s (1992) object file theory, if the pipe-pan information for $\mathrm{T} 1$ is overwritten by the random objects that intervene between $\mathrm{T} 1$ and $\mathrm{T} 2$, the object file will need to be modified in order to accommodate $\mathrm{T} 2$, and one might predict an $\mathrm{AB}$. The results are consistent with this notion of recency in object file reviewing and suggest that the locus of $\mathrm{AB}$ modulation in the full stream of random objects (Experiment 2) was the items intervening between the two targets, the randomobject masks, or a combination of the two.

\section{EXPERIMENT 5}

In Experiment 5, we examined the role of the randomobject items preceding T1. According to Raymond (2003), in this scenario, $\mathrm{T} 1$ should not be able to adopt the active object file without first making extensive modifications to the representation (because it is a different object). Consequently, T2 processing would suffer interference from $\mathrm{T} 1$ processing, and one would anticipate an $\mathrm{AB}$ effect. This experiment also uses a random-object mask. If the random-object mask alone is sufficient to have caused an $\mathrm{AB}$ in the full-stream condition (Experiment 2) and in the previous experiment, one might anticipate a significant effect here as well. This experiment was the same as Experiment 4, with the exceptions described below.

\section{Method}

Participants. Sixteen volunteers (11 females and 5 males; mean age, 21.5 years, $S D=2.63$ years) were recruited from either the community participant database (11) or the student participant panel (5).

Stimuli and Procedure. RSVP streams were the same as those in the previous experiment, except that 11 random objects were inserted before T1, rather than between the two targets. A random-object mask immediately followed T1 and T2. A typical RSVP stream is illustrated in Figure 11. As in the previous experiment, half of the 


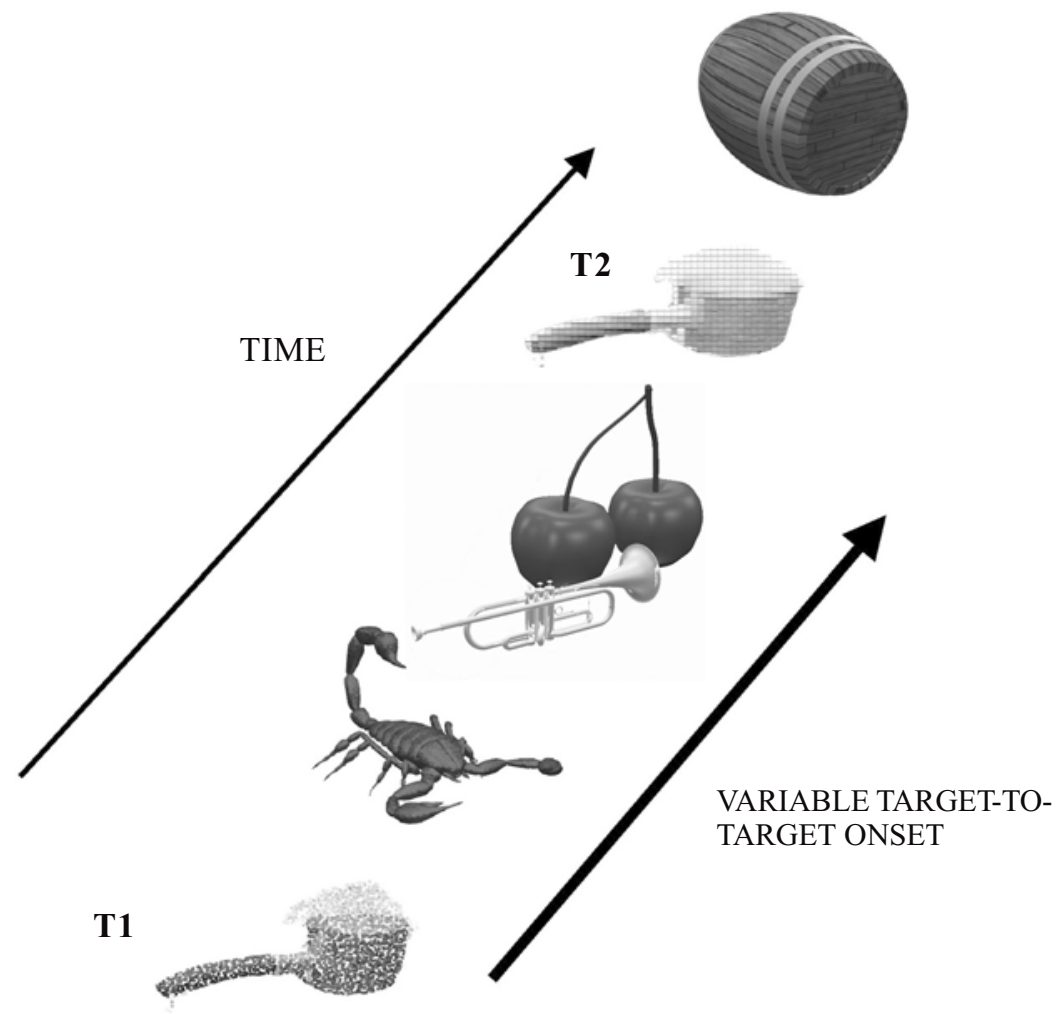

No items before $\mathrm{T} 1$

Figure 9. An example of the modified critical items streams used in Experiment 4. Streams were the same as those used in Experiment 3, except for the use of a randomobject mask and random objects intervening between $T 1$ and $T 2$. Depending on the serial position of $\mathrm{T} 2$, one to seven random objects were presented between $\mathrm{T} 1$ and $\mathrm{T} 2$. T2 is shown as occurring in Serial Position 4, which corresponds to a stimulus onset asynchrony of $267 \mathrm{msec}$.

participants completed their single and dual experimental trials in two sets, and the remaining 8 participants completed two sets of 140 trials (20 trials at each SOA).

\section{Results and Discussion}

The data from 1 participant were replaced because the participant made $51 \% \mathrm{~T} 2$ errors in the single-task condition. As in the previous experiment, prior to pooling all the data, the data from participants who had completed two sets of trials were analyzed. This analysis revealed that the data for Set 1 and Set 2 were not significantly different $[F(1,7)=3.343, p>.05]$. However, for consistency with Experiment 4, only Set 2 data were pooled with the single set data, and this reduced data set was used in all the subsequent analyses.

The mean percentage of correct $\mathrm{T} 1$ responses in the dual-task trials was $85 \%(S D=14.58 \%)$. Mean AB magnitudes were calculated, and these are displayed in Figure 12 , which suggests that there was no $\mathrm{AB}$. This was confirmed by a nonsignificant effect of SOA $[F(6,90)=$ $1.591, p>.05]$. Interestingly, 5 participants exhibited large order effects, in that their single-task performance was worse than their dual-task performance (yielding a number of negative values for nearly all the SOAs). Generally, counterbalancing the order of the single- and the dual-task trials will control for adverse effects of task order. Therefore, such an outcome may have been a function of the stream type. Given that all the individuals began with three sets of dual-task practice trials, it may have been difficult for these participants to subsequently ignore $\mathrm{T} 1$ if they had just completed either the dual-task trials ( 1 participant) or the three sets of dual-task practice trials (4 participants). To successfully achieve this outcome, the participants would need to attend to the first visual event that occurred after a variable blank interval (because there were no items presented between T1's mask and T2), and it may have been harder to attend to (or ignore) a variable blank offset than a number of random objects.

The results of this experiment contrast with those in the work of Raymond (2003). Given that there were pre-T1 items present and $\mathrm{T} 1$ was always a different object from 
A

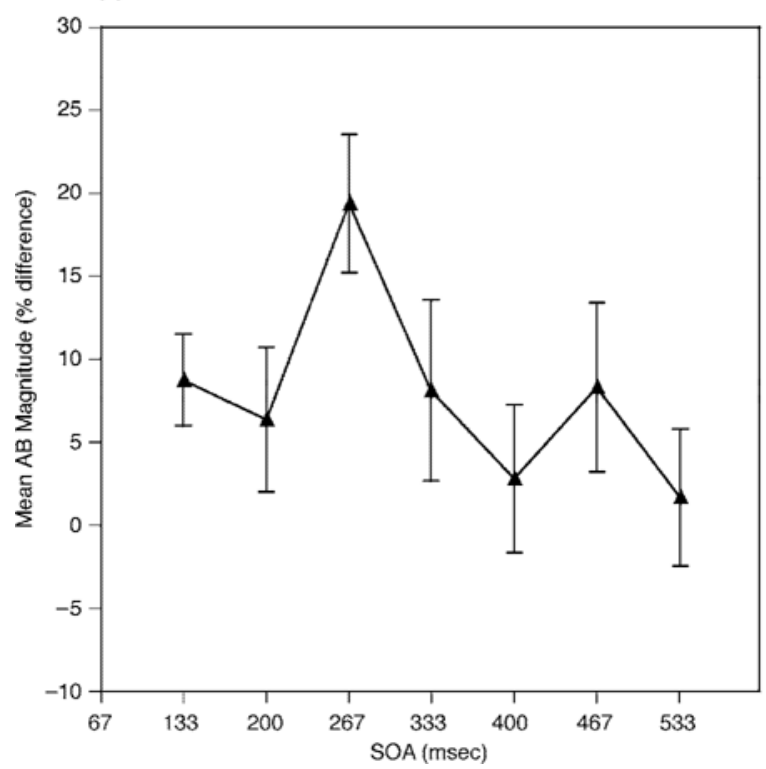

B

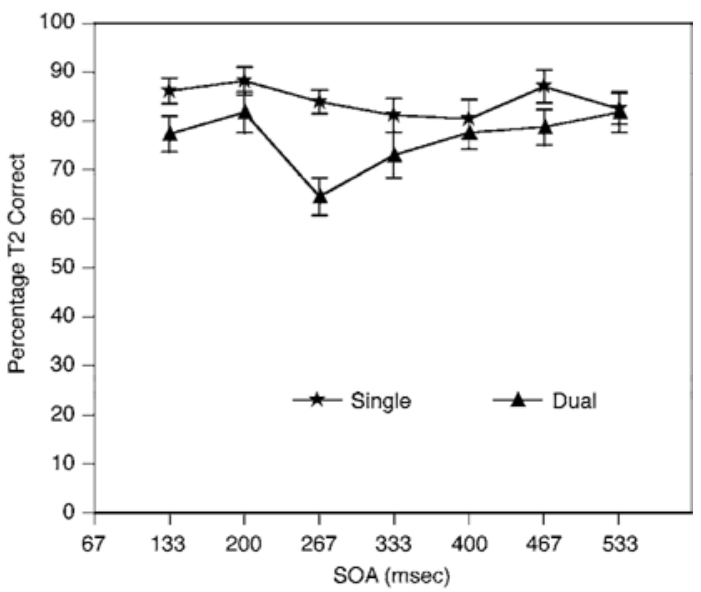

Figure 10. (A) Mean attentional blink (AB) magnitude for each stimulus onset asynchrony (SOA) for the modified critical items stream, which involved presenting a variable number of random objects between T1's mask and T2, as well as using a random object mask. Vertical bars represent standard error $( \pm 1)$. The same data are displayed in an alternative way in panel $B$, which depicts $T 2$ performance for both single- and dual-task trials.

the preceding items, one would predict an $\mathrm{AB}$ (although Raymond might have anticipated a shortened AB, because both $\mathrm{T} 1$ and $\mathrm{T} 2$ were the same object). However, a significant $\mathrm{AB}$ was not observed, suggesting that in the full stream of random objects (Experiment 2), the items preceding $\mathrm{T} 1$ played little, if any, role in modulating the $\mathrm{AB}$ effect. The results of this experiment raise problems for Kahneman et al.'s (1992) notion of recency in object file reviewing, which would predict that $\mathrm{T} 1$ 's pipe-pan information should be overwritten by its random-object mask. However, it might be true that a single random object was insufficient to completely overwrite the T1 information, especially since it would have been fully processed on virtually every trial.

In Experiments 4 and 5, we examined whether the locus of $\mathrm{AB}$ modulation in Experiment 2 was centered on the items that intervened between T1 and T2 (Experiment 4) or on the items that preceded T1 (Experiment 5). In both experiments, a random-object mask was used to match the original full-stream random-object experiment, which revealed a typical AB signature. In Experiment 4 , a significant $\mathrm{AB}$ was observed, suggesting that in the random-object full-stream condition (Experiment 2), the locus of $\mathrm{AB}$ modulation was the random objects that intervened between $\mathrm{T} 1$ and $\mathrm{T} 2$, the random-object mask, or a combination of the two. In Experiment 5, in contrast to the theoretical predictions based on the work of Raymond (2003), no AB effect was observed, suggesting that in the random-object full-stream condition (Experiment 2), the locus of $\mathrm{AB}$ modulation was not the items preceding $\mathrm{T} 1$ or the random-object mask.
An alternative explanation of the results in the first two experiments could be that they were due to differential effects of perceptual masking. If a random object is more efficient than a pipe-pan at masking $\mathrm{T} 1$, one might anticipate a robust $\mathrm{AB}$ in the full-stream random-object stream (Experiment 2) and an attenuated AB in the fullstream morph stream (Experiment 1). Therefore, Experiments 4 and 5 should both have yielded significant $A B$ effects, since a random-object mask was present in each case. Given that only Experiment 4 yielded an AB effect, one can conclude that the random objects intervening between $\mathrm{T} 1$ and $\mathrm{T} 2$ served to modulate the $\mathrm{AB}$ effect in the full-stream experiment. In the next experiment (Experiment 6), we examined masking in the previous experiments and introduced a new, very effective mask while attempting to equate masking across the three full-stream RSVP conditions: smooth morph, scrambled morph, and random objects.

\section{EXPERIMENT 6}

Experiment 6 marked a departure from critical items experiments and a return to the full-stream RSVP paradigm. As in the original full-stream experiments (Experiments 1 and 2), this experiment employed streams consisting of smooth morph, scrambled morph, or random-object images. Earlier, we claimed that the results of those experiments were due to differences in object file continuity. An alternative explanation relates to the efficacy of target masking. It is possible that a random object serves as a more efficient mask than does the mask used in the 


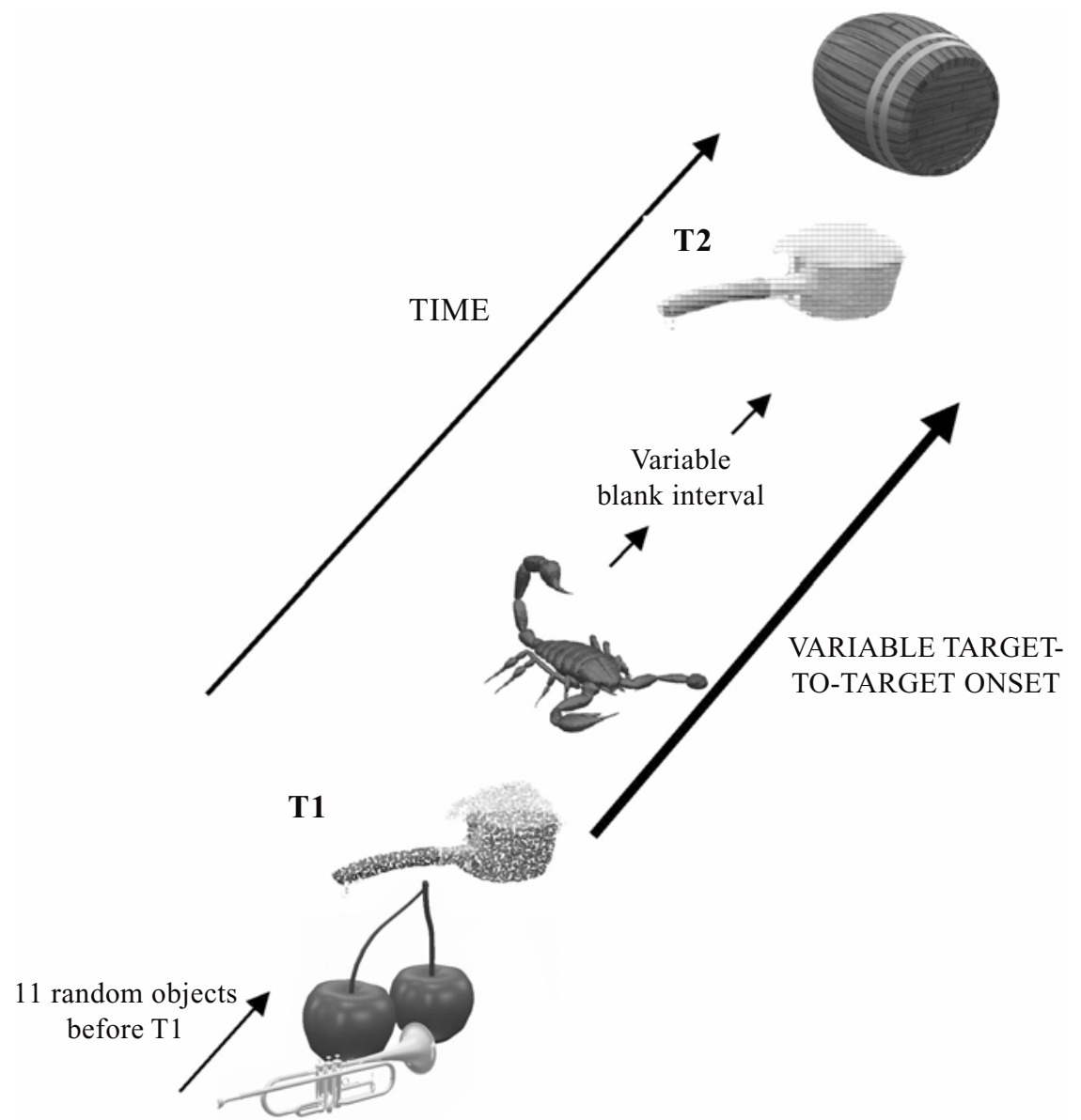

Figure 11. An example of the modified critical items streams used in Experiment 5. Streams were the same as those in Experiment 4, except that 11 random objects preceded T1 and only a single random object appeared after each target. A variable blank interval (depending on stimulus onset asynchrony occurred between T1's mask and T2.

smooth morph condition, which was the next consecutive pipe-pan. If this were true, the outcomes-no AB in the morph condition but a significant $\mathrm{AB}$ in the randomobject condition - would be predicted. However, the same argument does not hold for the scrambled morph condition, where a robust $\mathrm{AB}$ effect was obtained despite a pipe-pan mask chosen randomly from the remaining morph images having been used. With this in mind, the rationale underlying Experiment 6 was twofold. The first was to try to equate visual perceptual masking across all three streams, and the second was to ensure that the textured targets were adequately masked in all the conditions by using a textured mask.

If the results of Experiments 1 and 2 were due to differences in perceptual masking, then, when masking is effective as well as equated across all three stream types, one might expect a robust $\mathrm{AB}$ effect of equal magnitudes in all the conditions. Alternatively, if the results of the original full-stream experiments were due to object file related factors, one should observe outcomes similar to those obtained in Experiments 1 and 2-that is, a robust $\mathrm{AB}$ in the random-object stream, which represents substantial object file modifications, an attenuated $\mathrm{AB}$ in the smooth morph stream, in which minimal object file changes were required, and an intermediate $\mathrm{AB}$ effect in the scrambled morph stream, where the degree of object file modification was somewhere in between those required by the smooth morph and the random-object streams.

\section{Method}

This experiment was a replication of Experiments 1 and 2, with the following exceptions.

Participants. Thirty-six volunteers (26 females and 10 males; mean age, 23.3 years, $S D=4.83$ years) were recruited from either the community participant database (25) or the student participant panel (11).

Stimuli and Procedure. RSVP streams for this full-stream experiment were the same as those presented in Experiments 1 and 2, but in this experiment, the target masks were equated as much as possible across all types of stream: smooth morph, scrambled morph, and random objects. All the targets were masked by composite texture masks. These were created by editing the underlying pipe-pan image so that it consisted of a mixture of big and small dots, as well as big and small squares. Morph movie frames numbered 13-21 
A

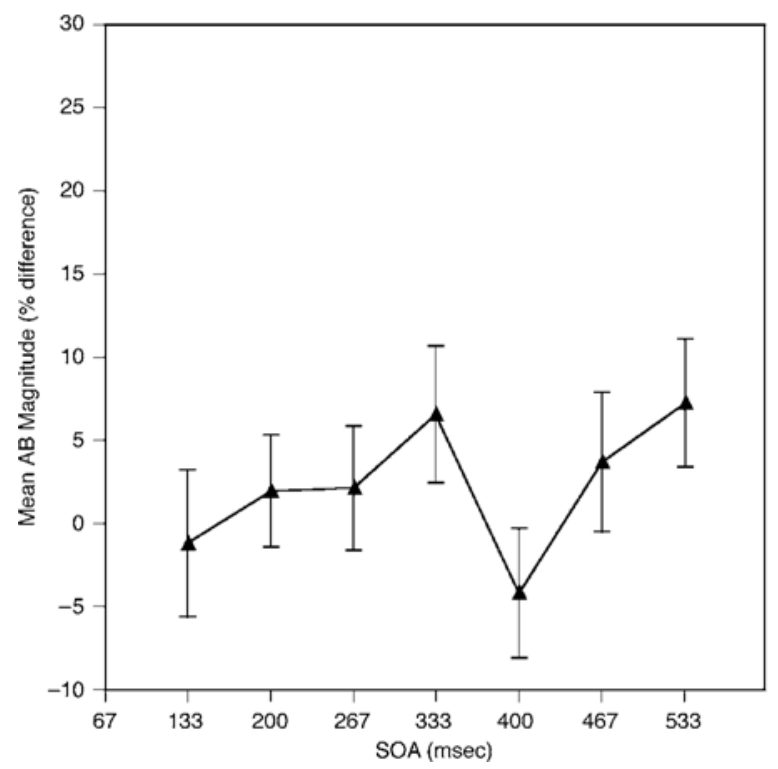

B

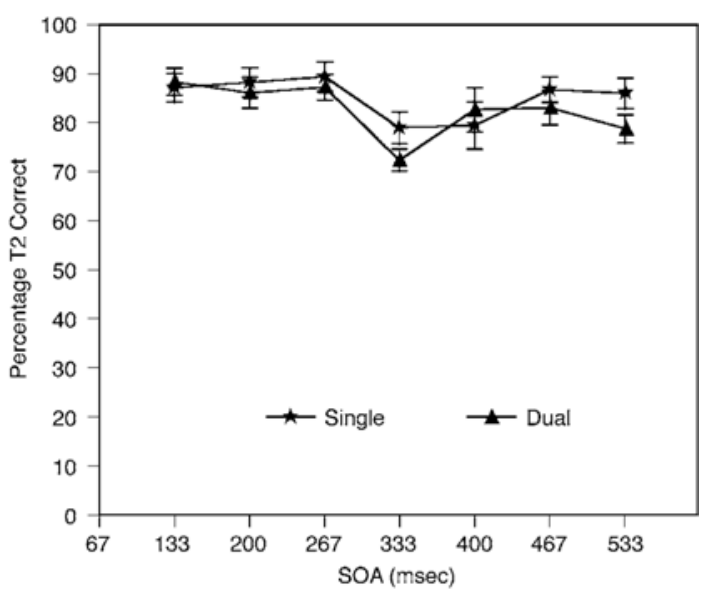

Figure 12. (A) Mean attentional blink (AB) magnitude for each stimulus onset asynchrony (SOA) for the modified critical items stream that involved presenting 11 random objects prior to $\mathrm{T} 1$, as well as using a random-object mask. Vertical bars represent standard error $( \pm 1)$. The same data are displayed in an alternative way in panel $B$, which depicts $T 2$ performance for both single- and dual-task trials.

were adapted in this way. Targets in the smooth morph condition were masked by a composite version of the next sequential pipe-pan image (i.e., when T2 was derived from the 15 th pipe-pan image, the mask for that target was derived from the 16th pipe-pan image).

The same composite texture masks (Morph Frames 13-21) were used to mask targets in the scrambled and random-object conditions, with the proviso that the mask was never the next consecutive item (in order to avoid creating a short morph episode in either condition). Target-mask combinations were identical in the scrambled and the random-object conditions (e.g., in the scrambled morph condition, if T1 [Frame 13] was masked by a composite version of Morph Frame 17 and T2 [Frame 15] was masked by a composite Frame 19, the same target-mask combination was used for the equivalent stream in the random-object condition). An example composite mask is shown in Figure 13, and a typical RSVP stream is illustrated in Figure 14.

As in the previous experiments, the participants completed three sets of practice trials, but at a slower rate. The first set comprised 16 trials ( 4 trials at each of T2 Serial Positions 5-8, corresponding to SOAs of $533,639,746$, and $853 \mathrm{msec}$ ) at the slower rate of $186.62 \mathrm{msec}$ per stimulus (five items per second). The second set

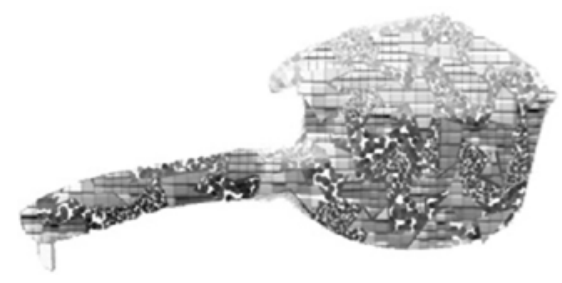

Figure 13. An example of a composite texture pipe-pan mask, which was made up of both big and small squares and dots. Masks were created for eight morph frames (13-21). comprised 12 trials ( 4 trials at each of T2 Serial Positions 2-4, corresponding to SOAs of 213,320 , and $427 \mathrm{msec}$ ) at the shorter duration of $159.96 \mathrm{msec}$ per item (six items per second). The third set of practice trials comprised 28 trials ( 4 trials at all SOAs) at a shorter duration of $106.64 \mathrm{msec}$ per stimulus, which corresponds to a presentation rate of nine items per second. One hundred forty experimental trials (20 trials for each of the seven serial positions of T2) were presented at a rate of nine items per second. All the participants completed their two sets of experimental trials (single- and dual-task trials) in two sets: Set 1 consisted of 56 trials (yielding 8 trials at each SOA), and Set 2 consisted of 84 trials (yielding 12 trials at each SOA).

\section{Results and Discussion}

In the scrambled morph condition, the data from 3 participants were replaced because they made excessive T2 errors $(54 \%$ and $66 \%)$ in the single-task trials or excessive T1 errors (54\%) in the dual-task trials. Similarly, in the random-object condition, the data from 5 participants were replaced because they made excessive T2 errors $(50 \%, 50 \%, 54 \%, 58 \%$, and $61 \%)$ in the single-task trials. In contrast, no data from the smooth morph condition needed to be replaced on the basis of T2 or T1 accuracy criteria.

Despite there being no significant difference of set across all stream types $[F(1,33)=3.343, p>.05]$, only the data from the second set ( 84 trials, 12 trials at each ISI) were used in all the subsequent analyses, to provide consistency with the previous experiments reported here (Experiments 4 and 5). The mean percentages of correct T1 responses in the dual-task trials were virtually identical for the smooth morph $(M=79 \%, S D=14.60 \%)$, 


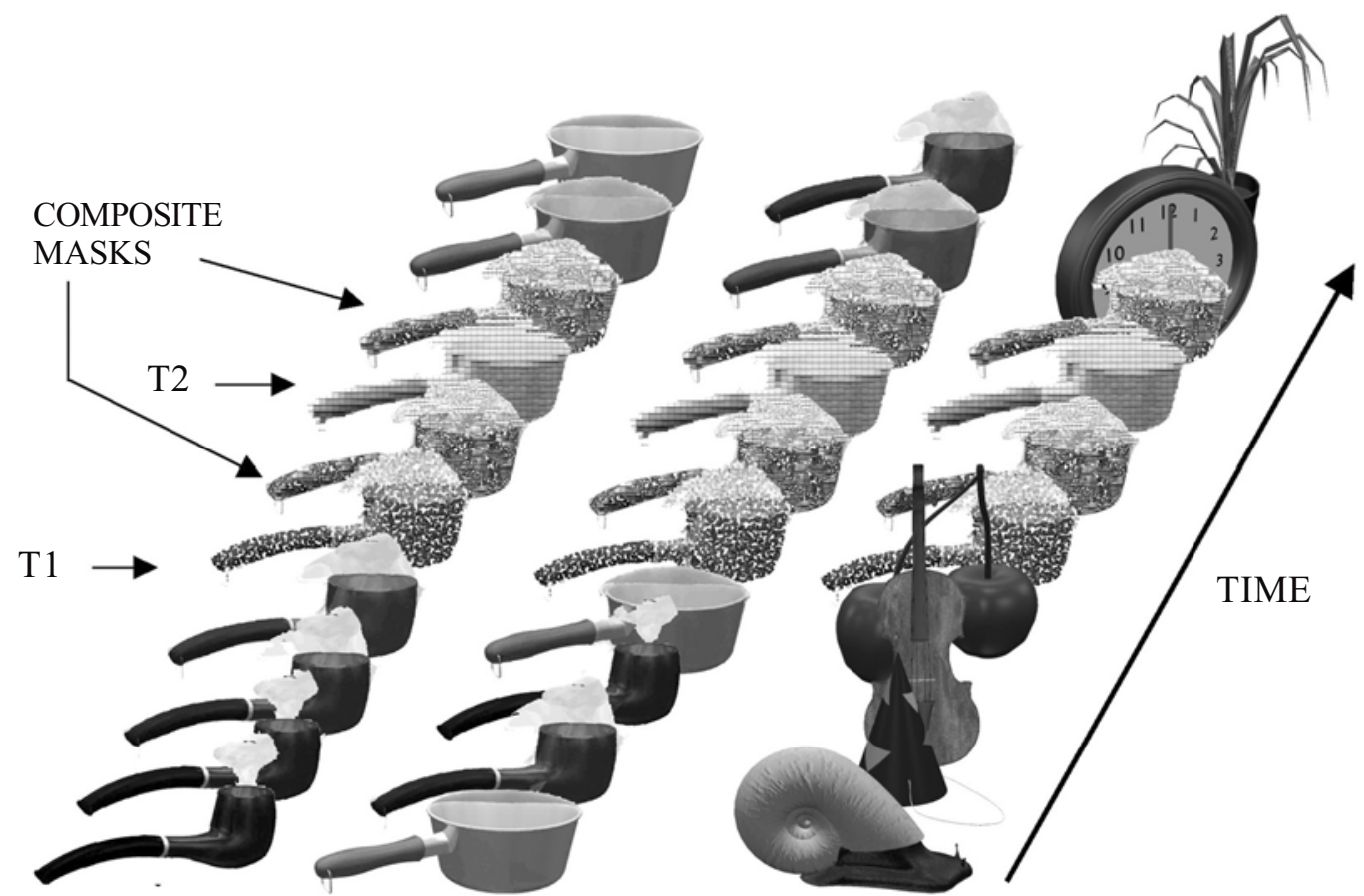

Figure 14. Example rapid serial visual presentation streams for the smooth morph (left), scrambled morph (center), and random-object (right) conditions in Experiment 6. Targets in the smooth morph condition were masked by a textured version of the next consecutive frame. Targets were never masked by a consecutive image in the scrambled or random-object conditions, where masks were randomly selected from the eight composite texture masks. The choice of masks was identical in the scrambled morph and the random-object conditions. Streams have been truncated for practical purposes.

scrambled morph $(M=80 \%, S D=16.09 \%)$, and randomobject $(M=78 \%, S D=18.22 \%)$ stream types. On the basis of boxplot analyses, the data for 3 participants were removed (1 from each group), reducing the overall sample size from 36 to 33 ( 11 in each group). Mean AB magnitudes were calculated for each stream type, and these are displayed in Figure 15 (panel A).

Figure 15 suggests that there was an SOA effect in each of the three conditions, although to different degrees. As was anticipated, there seems to have been a greater effect in the random-object condition than in the scrambled condition and a greater effect in the scrambled condition than in the smooth morph condition. Given that each stream type yielded at least some effect of SOA, it is not surprising that a nonsignificant interaction was obtained between stream type and SOA $[F(12,180)=$ $1.123, p>.05]$. The main effect of SOA was significant $[F(6,180)=3.709, p<.01]$, but the main effect of stream was not $[F(2,30)=2.005, p>.05]$.

This significant effect of SOA was again evident in a trend analysis, which confirmed there to be a global linear decrease in $\mathrm{AB}$ magnitude with increasing SOA $[F(1,30)=19.209, p<.01]$. The trend analysis revealed that the local changes in the slope of the linear trend were significantly different across the three stream types $[F(2,30)=4.923, p<.02]$. Trend analysis had been used previously in $\mathrm{AB}$ research (see, e.g., Brehaut et al.,
1999; Kawahara, Di Lollo, \& Enns, 2001; Shih, 2000). In order to unpack the global linear trend, additional trend analyses were also conducted for each stream type. These will now be presented alongside stream-by-stream comparisons between this experiment and the original full-stream experiments (Experiments 1 and 2). The role of the textured mask will then be considered.

Random-object stream. For the random-object stream, a significant linear trend was observed $[F(1,10)=$ $23.405, p<.02]$. Subsequent analyses (one-tailed $t$ tests) revealed significant $\mathrm{AB}$ magnitudes at $213 \mathrm{msec}[t(10)=$ $4.084, p<.01], 320 \mathrm{msec}[t(10)=4.921, p<.01]$, and $533 \mathrm{msec}[t(10)=3.418, p<.01]$. A nonsignificant outcome was obtained for the 427-msec SOA $[t(10)=1.211$, $p>.05]$; this outcome was, however, due to an extreme negative score, and as soon as 1 individual with a score of -25 was removed, the $p$ value for this analysis reached the $<.05$ level. Therefore, for the random-object stream, a robust $\mathrm{AB}$ effect was observed, and there was no recovery until $640 \mathrm{msec}$.

We have consistently seen the random-object fullstream result in an $\mathrm{AB}$ effect, irrespective of whether targets were masked by a nontextured random object (as in Experiment 2) or by a composite textured pipe-pan image (Experiment 6). Furthermore, an ANOVA $^{1}$ has shown there to be no statistical difference in $\mathrm{AB}$ magnitude across SOAs between the two experiments $[F(3,51)=$ 
A

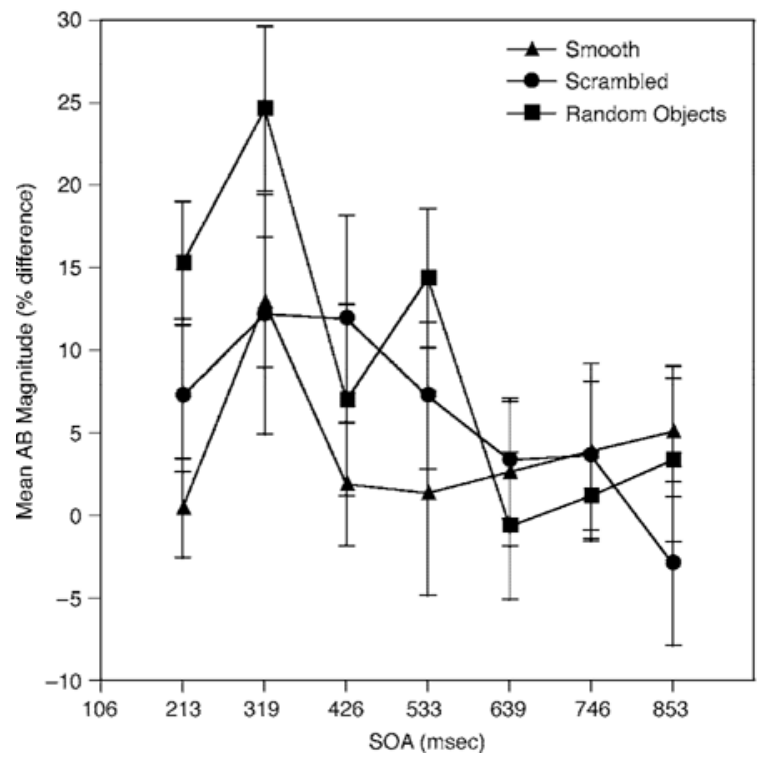

B

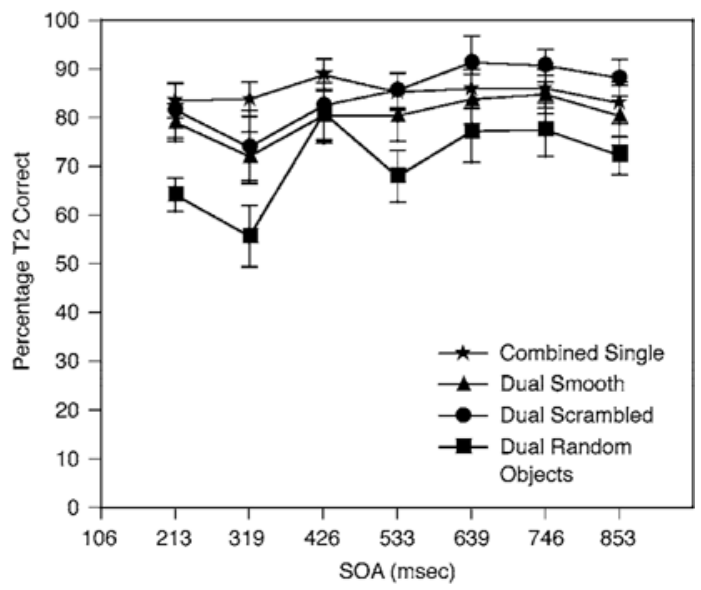

Figure 15. (A) Mean attentional blink (AB) magnitude for each stimulus onset asynchrony (SOA) for smooth morph, scrambled morph, and random-object conditions. In this full-stream experiment, T1 and T2 were masked by composite texture masks. Standard error $( \pm 1)$ is represented by the vertical bars. The same data are displayed in an alternative way in panel $B$, which depicts $\mathrm{T} 2$ performance for smooth morph, scrambled morph, and random-object streams for the dual-task trials. The single-task baseline reflects combined performance in the ignore-T1 condition for the smooth morph, scrambled morph, and random-object streams.

1.998, $p>.10$ ]. In Experiment 2, lag-1 sparing was observed and discussed. In contrast, the significant linear trend in the present experiment suggests that lag-1 sparing was not present. Although the differences in timing parameters make it impossible to make direct comparisons between the two experiments, it is interesting to note that at around $200 \mathrm{msec}$ (i.e., the third serial position in Experiment 2 and the first serial position in Experiment 6), comparable $\mathrm{AB}$ magnitudes were observed.

The scrambled morph stream. The trend analysis conducted on the scrambled morph stream approached significance $[F(1,10)=4.819, p<.06]$, thus demonstrating less of an SOA effect than the random-object stream. One-tailed $t$ tests were conducted for the first four SOAs in order to determine whether the mean $\mathrm{AB}$ magnitude was significant at a given SOA. These analyses revealed that the $427-\mathrm{msec}$ SOA was significant at the .05 level $[t(10)=1.904, p<.05]$, whereas the other three SOAs approached significance at the same level: $213 \mathrm{msec}[t(10)=1.574, p>.05], 320 \mathrm{msec}[t(10)=$ $1.681, p>.05]$, and $533 \mathrm{msec}[t(10)=1.637, p>.05]$. A great deal of variability was observed in the scrambled morph group, and this, coupled with the relatively small $\mathrm{AB}$ magnitudes, accounts for the results of the analyses for this group. However, there was evidence that an AB effect was present and that it endured until $640 \mathrm{msec}$.

A comparison of the outcomes for the scrambled morph stream in this experiment and Experiment 1 reveals that the patterns of results were qualitatively very similar.
Whereas in Experiment 1 there was a robust $\mathrm{AB}$ effect, such an effect was only marginally significant here, probably because of increased variability. However, an ANOVA conducted on the two sets of data showed there to be no significant difference in $\mathrm{AB}$ magnitude across SOAs between the two groups $[F(3,51)=0.385, p>.10]$.

The smooth morph stream. In contrast to the other two stream types, the trend analysis conducted for the smooth morph was nonsignificant $[F(1,10)=0.068$, $p>.05]$, suggesting that, overall, there was no SOA effect. However, a significant degree of $\mathrm{AB}$ magnitude was revealed for the 320-msec SOA $[t(10)=3.266, p<.01]$, but the same was not true for the $213-\mathrm{msec} \operatorname{SOA}[t(10)=$ $0.151, p>.05]$, the $427-\mathrm{msec}$ SOA $[t(10)=0.513, p>$ $.05]$, or the 533-msec SOA $[t(10)=0.220, p>.05]$. On the basis of these results, it is claimed that $\mathrm{AB}$ attenuation was observed in the smooth morph condition, with the exception of the 320-msec SOA.

$\mathrm{AB}$ attenuation was also observed in the original fullstream morph experiment (Experiment 1). An ANOVA showed there to be no difference in $\mathrm{AB}$ magnitude across SOAs between Experiment 1 and the present experiment $[F(3,51)=1.952, p>.10]$. A qualitative analysis of both experiments revealed a similar increase in $\mathrm{AB}$ magnitude for the second serial position of T2 (although these corresponded to different SOAs), although this effect was smaller in Experiment 1. The results of both experiments demonstrate that $\mathrm{AB}$ attenuation is observed when the RSVP stream uses a single object file through- 
out. Furthermore, this effect cannot be due to inadequate visual perceptual masking, since an adequate mask was used in Experiment 6.

T1 performance and the composite texture mask. T1 performance for the smooth and the scrambled morph full streams decreased to $79 \%$ and $80 \%$, respectively, when the target was masked by a composite textured pipe-pan image (in Experiment 6), as compared with when it was masked by a nontextured pipe-pan image (in Experiment 1 ), where $\mathrm{T} 1$ performance was at $97 \%$ and $89 \%$, respectively. This suggests that the new, composite texture pipe-pan mask did serve as a more effective mask for a pipe-pan target in streams consisting entirely of pipe-pan items, whether they were presented sequentially or not. This is supported by the fact that $\mathrm{T} 1$ performance was lower in Experiment 6 despite a far slower presentation rate having been used, although this reduced $\mathrm{T} 1 \mathrm{accu}-$ racy may have resulted from resource-limiting factors, rather than from encoding-difficulty-related factors (McLaughlin, Shore, \& Klein, 2001).

For the random-object full stream, for which T1 was masked by a nontextured random object (Experiment 2), T1 performance was at $87 \%$. Where the same target was masked by a textured pipe-pan (Experiment 6), T1 performance was seen to decrease slightly to $81 \%$. This suggests that in both experiments, despite differences in the physical nature of the mask, visual perceptual masking was about the same.

These differences in masking can be explained if one considers the perceptual nature of the streams. For both morph groups, detection of the textured target involves searching among many similar but nontextured pipe-pan items. Adding a texture to the mask makes the task more difficult, because the featural nature of the target (the dotted texture) is no longer distinct. One might, therefore, anticipate a reduction in target detection. Now let us apply the same rationale to the random-object stream. Again, when the mask is a nontextured random object, the target is featurally distinct on two counts. First, as for the morph streams, it is the only item in the stream made up of dots. Second, there are only two pipe-pans in the stream (which may constitute more of a pop-out search), and both of them are targets. In the random-object stream, viewers know that if the item is a pipe-pan, it is relevant to the task. However, when a textured pipe-pan mask is used, this strategy is less useful, and one might anticipate some reduction in target accuracy.

In Experiment 6, the same combination of target and mask was used for the random-object and scrambled morph streams. Therefore, if it is the four critical items that determine the magnitude and shape of the $\mathrm{AB}$, identical outcomes should have been observed for these two stream types. Whereas T1 performance was about the same, the magnitude and shape of the $\mathrm{AB}$ functions were not. This outcome lends support to the notion that other items in the stream affected the $\mathrm{AB}$ outcome observed.

Concluding comments. The outcomes of Experiment 6 are in agreement with an intuitive analysis of Fig- ure 15 and the experimental predictions. The randomobject stream, in which it is proposed that object file continuity is maximally disrupted, led to a robust SOA effect. The scrambled object stream, in which it is supposed that object file continuity is less disrupted than in the random-object condition, did result in lower $\mathrm{AB}$ magnitudes, and statistical analyses were only marginally significant. On the other hand, the smooth morph condition, for which it is proposed that a minimal object file updating is required, led to almost a complete attenuation of the AB. These results mirror those reported in the first two experiments and confirm that those results were not due to differences in visual perceptual masking but were due to object file related factors.

\section{GENERAL DISCUSSION}

In these experiments, we set out to investigate the relationship between object file continuity and the AB. The full-stream experiments manipulated the degree of object file continuity, so that targets in an RSVP stream could or could not be easily linked to the same object file. The part-stream experiments were designed to identify what aspect of the full-stream paradigm modulates object file continuity and the AB. Here, we will attempt to synthesize the results of these experiments, beginning with a brief outline of all six experiments.

\section{Summary of Empirical Work}

The original full-stream RSVP experiments (Experiments 1 and 2) manipulated object file continuity or discontinuity so that $\mathrm{T} 1$ (a pipe-pan image made up of big or small dots) and T2 (a pipe-pan image made up of big or small squares) could or could not share the same object file. Experiment 1 used a morph stream that was presented either sequentially in RSVP (smooth morph) or randomly (scrambled morph). In the smooth morph condition, object file continuity was preserved, and the $\mathrm{AB}$ was attenuated. In the scrambled condition, a short-lived AB effect (recovery occurred by $267 \mathrm{msec}$ ) was obtained, reflecting some disruption of object file continuity. Experiment 2 used a full stream of random objects and yielded a robust $\mathrm{AB}$ effect that did not recover until $467 \mathrm{msec}$, reflecting a greater degree of object file modification.

Variations of the critical items $(\mathrm{T} 1, \mathrm{~T} 1+1, \mathrm{~T} 2$, and $\mathrm{T} 2+1$ ) paradigm were used in Experiments 3, 4, and 5 in order to investigate factors that modulated the $\mathrm{AB}$ outcomes in the full-stream smooth morph (where there was no $\mathrm{AB}$ ) and random-object (where there was a robust $\mathrm{AB}$ ) conditions. Experiment 3 utilized a pure critical items paradigm (consisting of the pipe-pan targets and their respective pipe-pan masks), and AB attenuation was again observed, suggesting that in the full morph stream the process of morphing was not a necessary condition to provide object file continuity. However, the lower level of variability in the data for the full-stream morph condition suggests that the morphing process did provide some additional degree of object file continuity. 
Experiment 4 utilized streams consisting of the two pipe-pan targets, random-object masks, and a variable number of random objects between T1's mask and T2. Despite high levels of variability, a significant AB was obtained overall, suggesting that the intervening stream items between $\mathrm{T} 1$ and $\mathrm{T} 2$ play a role in object file continuity and $\mathrm{AB}$ modulation in the original full stream of random objects. An alternative explanation is that the outcome in both cases (full stream and Experiment 4) was due to using a random-object mask. Prior to investigating the role of the mask, in Experiment 5, we examined the role of the random objects that appeared before $\mathrm{T} 1$ in the full-stream condition and utilized a modified critical items paradigm consisting of the two pipe-pan targets, random-object masks, and 11 random objects preceding T1. Contrary to theoretical predictions (i.e., Raymond, 2003), no AB effect was observed. This suggests that items preceding $\mathrm{T} 1$ played little, if any, role in $\mathrm{AB}$ modulation/object file continuity in the full stream of random objects (Experiment 2). This outcome also demonstrates that $\mathrm{AB}$ modulation in Experiment 2 was not simply due to the use of a random-object mask. Experiment 6 returned to using a full RSVP stream to examine visual perceptual masking and equate masking across the three stream types: smooth morph, scrambled morph, and random. A composite textured mask was used to ensure mask efficacy. Trend analyses revealed $\mathrm{AB}$ attenuation in the smooth morph condition, a marginally significant $\mathrm{AB}$ in the scrambled condition, and a significant $\mathrm{AB}$ in the random-object condition. These results replicate the original full-stream experiments (Experiments 1 and 2) and demonstrate that these outcomes are not due to differences in masking.

\section{The Main Experimental Outcomes and Their Implications}

The results of the full-stream RSVP experiments demonstrate there to be a link between object file continuity and modulation of the AB. That is, there is an inverse linear relationship between $\mathrm{AB}$ magnitude and the degree of object continuity inherent in the stream. We have shown that when RSVP targets can be linked to a common object file through the process of morphing, the $\mathrm{AB}$ is largely attenuated. Conversely, when object file continuity has been disrupted (as in the scrambled morph full stream) but the object file can be updated and used, rather than discarded and a new one generated, less AB attenuation is obtained, as compared with the smooth morph condition, in which few object file amendments are required and object continuity remains intact. At the other extreme, the full stream of random objects served to violate object file continuity to the degree that targets could not share the same object file without making extensive modifications to the object file or discarding the object file and generating a new one (possibly, whichever is most efficient). Consequently, such extensive object file processes corresponded with a loss of perceptual continuity and a robust $\mathrm{AB}$ effect. Rather than propose three separate categories of object file continuity (same file, update file, and new file), it seems better to assume a continuum whereby the degree of object file continuity relates to $\mathrm{AB}$ magnitude.

The differences in $A B$ magnitude across the three types of stream were not due to differences in visual perceptual masking. This was supported by Experiment 6, in which the results of the original full-stream experiments were replicated, despite visual perceptual masking having been equated across the three stream types. In addition, the scrambled and random-object streams in the same experiment had identical combinations of targets and masks, yet resulted in different outcomes. This suggests that the shape and duration of the $\mathrm{AB}$ are not determined solely by the critical items but are more likely due to object file related factors.

Having revealed a relationship between the $\mathrm{AB}$ and object file continuity, it was important to determine what these factors were - that is, what aspects of the full streams facilitated object file continuity and $\mathrm{AB}$ modulation. Three possible candidate explanations were considered: the process of morphing, the items preceding $\mathrm{T} 1$ (as was suggested by Raymond, 2003), or the intervening stream items between T1 and T2 (which concurs with Kahneman et al.'s, 1992, notion of recency in object file reviewing). All three candidate explanations could account for the results of the full-stream experiments. Consequently, part-stream experiments were crucial in investigating these alternatives. The major outcomes of these experiments will now be considered, along with relevant theoretical implications.

In these experiments, the process of morphing - the presentation of a complete sequence of pictures depicting one object smoothly changing into another-was found not to be a necessary condition in determining object file continuity (although its presence was associated with less variability and greater perceptual continuity). Kahneman et al.'s (1992) notion of recency in object file reviewing states that during the process of object file reviewing, the system has access only to the most recent object information (i.e., that pertaining to the visual item immediately preceding the current one). Hence, when one removes nearly all of the picture sequence that depicts object change (i.e., the process of morphing), the information that remains is entirely consistent with the concept of recency. This is consistent with object file continuity's having been preserved when the four pipe-pan critical items were presented alone (as in Experiment 3). The finding that the entire morphing sequence was not critical in providing object file continuity suggests that Raymond's (2003) use of motion may not have been crucial either. If so, it should be possible to adopt a critical items version of Raymond's experiment (trident targets and trident masks) and obtain object file continuity and $\mathrm{AB}$ attenuation.

Raymond (2003) claimed that the locus of object file continuity is the items preceding T1. In Raymond's series of trident experiments, she proposed that the first 
distractor trident in the stream generates an object file and that this file is subsequently adopted by $\mathrm{T} 1$ when it is the same object (a trident). However, when T1 is a different object, this necessitates changes to be made to the object file. Because the process of opening a new object file is attentionally demanding, this will have a detrimental effect on subsequent object file operations (such as those required in order to process T2). In the present experiments, evidence arising from the part-stream, preT1 experiment (Experiment 5) did not support Raymond's theoretical interpretation. These results suggest that, at least in the random-object full stream, the items preceding $\mathrm{T} 1$ played little role in modulating the $\mathrm{AB}$ effect. However, given the methodological differences between this work and that of Raymond (e.g., she used apparent motion rather than object change), the role of the pre-T1 items should not be completely eliminated.

Experimental evidence suggests that the intervening stream items between $\mathrm{T} 1$ and $\mathrm{T} 2$ provide the locus for object file continuity and $\mathrm{AB}$ modulation. A robust $\mathrm{AB}$ effect was obtained when the pipe-pan targets (with randomobject masks) were presented with other random objects intervening between T1 and T2 (Experiment 4). On the basis of this outcome, one can claim that (at least for the full stream of random objects) the stream items that intervened between $\mathrm{T} 1$ and $\mathrm{T} 2$ were responsible for object file continuity and AB modulation. One can draw upon Kahneman et al.'s (1992) concept of recency in object file reviewing in order to conceptualize the way in which the intervening items might be implicated in object file continuity. When the visual information for the pipe-pan $\mathrm{T} 1$ is overwritten by the intervening random objects, $\mathrm{T} 1$ and T2 can no longer share the same object file until substantial modifications have been made to it. Furthermore, the degree of object file discontinuity determines the extent to which the object file needs to be modified and the resources required. These operations are, consequently, manifest in observed $\mathrm{AB}$ magnitudes.

Grandison, Ghirardelli, and Egeth (1997) claimed that any +1 item that serves as an effective mask will lead to an $\mathrm{AB}$ effect. However, the smooth morph condition in Experiment 6 raises problems for this claim, because $A B$ attenuation was observed despite the presence of an effective mask. The same condition also provides counterevidence to Shapiro et al. (1994), who argued that in order for the $\mathrm{AB}$ to occur, attention must be directed to an object or a patterned target stimulus.

McAuliffe and Knowlton (2000) claimed that AB magnitude is determined by featural complexity of the +1 item (rather than conceptual similarity or spatial area), and their work revealed a linear relationship between $A B$ magnitude and the featural similarity of $\mathrm{T} 1$ and the +1 item. However, the results of Experiment 6, in which different $A B$ magnitudes were obtained across all three stream types despite no change to the featural (or conceptual) similarity of the +1 item, show that featural complexity cannot be the only factor that contributes to $\mathrm{AB}$ modulation.
These experiments also demonstrate that $\mathrm{AB}$ magnitude is not determined simply by interference arising from the +1 item but that other stream items are also implicated (Chun \& Potter, 1995; Isaak, Shapiro, \& Martin, 1999). On the basis of the part-stream experiments, the most likely candidates are the items that intervene between T1's mask and T2. Although the nature of such interference may be featural (or conceptual, if we conceptualize pipes, pans, and random objects as different categories), the results of the present series of experiments suggest that object file related factors are heavily implicated.

Duncan et al. (1994) established that it is possible to obtain an $\mathrm{AB}$ by using only the critical items. However, although their work demonstrates that an $\mathrm{AB}$ can be obtained without the remaining stream items, it does not eliminate the possibility that, when present, distractor items may be determinants of $\mathrm{AB}$ modulation. Our research presents the first attempt to investigate the role of distractors in terms of implicating the stream items that intervene between $\mathrm{T} 1$ and $\mathrm{T} 2$ in $\mathrm{AB}$ modulation, as well as object file continuity.

Another issue to consider is that the different outcomes for the full-stream experiments are due to differences in the degree of visual transience inherent in the visual displays (i.e., differences in offsets and onsets of RSVP items). In the smooth stream (where the AB was attenuated), there was virtually no visual transience in the stream, as compared with the random-object stream (where a robust $\mathrm{AB}$ effect was obtained), which was characterized by a high level of visual transience. Initially, such an explanation does appear plausible. However, the same argument will not apply to the scrambled morph stream. When creating the morph, it was essential to select items that were very similar in terms of size and shape, in order to obtain a smooth process of change between the objects. Therefore, when the morph was subsequently scrambled, there was a relatively small degree of transience present across successive frames. Similarly, at least for the +1 item, the overall spatial area (and therefore, visual transience) has been found to be unimportant in determining AB magnitude (McAuliffe \& Knowlton, 2000). In light of these points, an alternative explanation in terms of visual transience is not compelling. In addition, in the real world, different objects correspond to a variety of spatial configurations. Therefore, it may be problematic, as well as less ecologically valid, to manipulate object file discontinuity while totally eliminating visual transience.

The $\mathrm{AB}$ attenuation in the smooth morph condition has implications for two of the central tenets identified by Shapiro et al. (1997) as being common to the major $\mathrm{AB}$ theoretical positions. The first tenet was that greater resources are required to process $\mathrm{T} 1$ to the level of report when it is adequately masked. The second held that because attentional resources are finite, the increased processing requirement for $\mathrm{T} 1$ will result in fewer resources being available to process $\mathrm{T} 2$. This, in turn, will lead to the decay or substitution of $\mathrm{T} 2$, because it has not been 
able to attain a durable representation. On the basis of the smooth morph results presented here, perhaps one might offer a caveat to these tenets: When object file continuity is present, both target tasks may be processed as part of the same attentional episode in which one object is seen to change from one state to another, rather than one object being replaced by another.

Our results suggest that the object file, however neurally implemented, may serve the important function of maintaining object constancy in the face of an ever changing perceptual world by reducing the demand on attention typically required to monitor changing perceptual objects and events. Such a mechanism would almost certainly play an extremely important role in human behavior, insofar as it provides a means for conserving attention when seemingly endless demands are placed upon this limited resource. Given the complexity of the perceptual world in which humans must operate, any mechanism enabling them to monitor a dynamic environment without attentional cost must be viewed as an essential part of their perceptual "tool kit."

\section{REFERENCES}

Baylis, G. C., \& Driver, J. (1993). Visual attention and objects: Evidence for hierarchical coding of location. Journal of Experimental Psychology: Human Perception \& Performance, 19, 451-470.

Brehaut, J. C., EnNS, J. T., \& Di Lollo, V. (1999). Visual masking plays two roles in the attentional blink. Perception \& Psychophysics, 61, 1436-1448.

Broadbent, D. E., \& Broadbent, M. H. P. (1987). From detection to identification: Response to multiple targets in rapid serial visual presentation. Perception \& Psychophysics, 42, 105-113.

Chun, M. M., \& PotTer, M. C. (1995). A two-stage model for multiple target detection in rapid serial visual presentation. Journal of Experimental Psychology: Human Perception \& Performance, 21, 109-127.

DUNCAN, J. (1984). Selective attention and the organization of visual information. Journal of Experimental Psychology: General, 113, 501517.

Duncan, J., Ward, R., \& Shapiro, K. L. (1994). Direct measurement of attentional dwell time in human vision. Nature, 369, 313-315.

EnNS, J. T., \& Di Lollo, V. (1997). Object substitution: A new form of masking in unattended visual locations. Psychological Science, $\underline{\mathbf{8}}_{2}$ 135-139.

GiESBRECHT, B., \& Di Lollo, V. (1998). Beyond the attentional blink: Visual masking by object substitution. Journal of Experimental Psychology: Human Perception \& Performance, 24, 1454-1466.

GoRDON, R. D., \& IRWIN, D. E. (1996). What's in an object file? Evidence from priming studies. Perception \& Psychophysics, 58, 1260-1277.

Grandison, T. D., Ghirardelli, T. G., \& Egeth, H. E. (1997). Beyond similarity: Masking of the target is sufficient to cause the attentional blink. Perception \& Psychophysics, 59, 266-274.

IsAAK, M. I., ShaPIRO, K. L., \& MARTIN, J. (1999). The attentional blink reflects retrieval competition among multiple rapid serial visual presentation items: Tests of an interference model. Journal of Experimental Psychology: Human Perception \& Performance, 25, 1774-1792.

Kahneman, D., Treisman, A., \& GibBS, B. J. (1992). The reviewing of object files: Object-specific integration of information. Cognitive Psychology, 24, 175-219.

KAWAhara, J.-I., Di Lollo, V., \& EnNs, J. T. (2001). Attentional re- quirements in visual detection and identification: Evidence from the attentional blink. Journal of Experimental Psychology: Human Perception \& Performance, 27, 969-984.

LAWRENCE, D. H. (1971). Two studies of visual search for word targets with controlled rates of presentation. Perception \& Psychophysics, 10, 85-89.

MCAuliffe, S. P., \& KNowlton, B. J. (2000). Dissociating the effects of featural and conceptual interference on multiple target processing in rapid serial visual presentation. Perception \& Psychophysics, $\underline{\mathbf{6 2}}_{2}$ 187-195.

MCLAUGHLIN, E. N., Shore, D. I., \& Klein, R. M. (2001). The attentional blink is immune to masking-induced data limits. Quarterly Journal of Experimental Psychology, 54A, 169-196.

RAYMOND, J. E. (2003). New objects, not new features, trigger the attentional blink. Psychological Science, 14, 54-59.

Raymond, J. E., SHAPIRO, K. L., \& ARneLL, K. M. (1992). Temporary suppression of visual processing in an RSVP task: An attentional blink? Journal of Experimental Psychology: Human Perception \& Performance, 18, 849-860.

REEVES, A., \& SPERLING, G. (1986). Attention gating in short-term visual memory. Psychological Review, 94, 180-206.

SeIfFert, A. E., \& Di Lollo, V. (1997). Low-level masking in the attentional blink. Journal of Experimental Psychology: Human Perception \& Performance, 23, 1061-1073.

Shapiro, K. L., ArNeLl, K. M., \& Raymond, J. E. (1997). The attentional blink. Trends in Cognitive Sciences, 1, 291-296.

ShapIRO, K. L., \& RAYMOND, J. E. (1994). Temporal allocation of visual attention. In D. Dagenbach \& T. H. Carr (Eds.), Inhibitory processes in attention, memory, and language (pp. 151-188). New York: Academic Press.

Shapiro, K. L., Raymond, J. E., \& Arnell, K. M. (1994). Attention to visual pattern information produces the attentional blink in rapid serial visual presentation. Journal of Experimental Psychology: Human Perception \& Performance, 20, 357-371.

SHIH, S.-I. (2000). Recall of two visual targets embedded in RSVP streams of distractors depends on their temporal and spatial relationship. Perception \& Psychophysics, 62, 1348-1355.

SPerling, G., \& WeichSElgartner, E. (1995). Episodic theory of the dynamics of spatial attention. Psychological Review, 102, 503-532.

Visser, T. A. W., Bischof, W. F., \& Di Lollo, V. (1999). Attentional switching in spatial and nonspatial domains: Evidence from the attentional blink. Psychological Bulletin, 125, 458-469.

Watson, S. E., \& Kramer, A. F. (1999). Object-based visual selective attention and perceptual organization. Perception \& Psychophysics, 61, 31-49.

WEICHSELGARTNER, E., \& SPERLING, G. (1987). Dynamics of automatic and controlled visual attention. Science, 238, 778-780.

\section{NOTE}

1. It was not possible to directly compare the results from Experiments 1 and 2 with those from Experiment 6. First, in Experiments 1 and 2, the first serial position of $\mathrm{T} 2$ was the $\mathrm{T} 1+1$ position, whereas in Experiment 6 this position was always occupied by the texture mask. Second, in Experiment 6, presentation rates different from those in Experiment 1 and, therefore, different SOAs, were used. Consequently, the longest SOA in Experiments 1 and 2 was 534 msec (just outside the AB range), whereas the longest SOA in Experiment 6 was $853 \mathrm{msec}$ (well outside the $\mathrm{AB}$ period). For these reasons, the first $\mathrm{SOA}$ for Experiments 1 and 2, and the last three SOAs from all the experiments was omitted from these stream-by-stream analyses across experiments.

(Manuscript received December 17, 2002; revision accepted for publication September 12, 2003.) 